\title{
Comparison of Birkeland current observations during two magnetic cloud events with MHD simulations
}

\author{
H. Korth ${ }^{1}$, B. J. Anderson ${ }^{1}$, J. G. Lyon ${ }^{2}$, and M. Wiltberger ${ }^{3}$ \\ ${ }^{1}$ The Johns Hopkins University, Applied Physics Laboratory, Laurel, MD, USA \\ ${ }^{2}$ Dept. of Physics and Astronomy, Dartmouth College, Hanover, NH, USA \\ ${ }^{3}$ High Altitude Observatory, National Center for Atmospheric Research, Boulder, CO, USA
}

Received: 19 March 2007 - Revised: 20 December 2007 - Accepted: 17 January 2008 - Published: 26 March 2008

\begin{abstract}
Low altitude field-aligned current densities obtained from global magnetospheric simulations are compared with two-dimensional distributions of Birkeland currents at the topside ionosphere derived from magnetic field observations by the constellation of Iridium satellites. We present the analysis of two magnetic cloud events, 17-19 August 2003 and 19-21 March 2001, where the interplanetary magnetic field (IMF) rotates slowly $\left(\sim 10^{\circ} / \mathrm{h}\right)$ to avoid time-aliasing in the magnetic perturbations used to calculate the Birkeland currents. In the August 2003 event the IMF rotates from southward to northward while maintaining a negative IMF $B_{y}$ during much of the interval. During the March 2001 event the IMF direction varies from dawnward to southward to duskward. We find that the distributions of the Birkeland current densities in the simulations agree qualitatively with the observations for northward IMF. For southward IMF, the dayside Region- 1 currents are reproduced in the simulation, but appear on average $5^{\circ}$ further poleward of their observed location, while the nightside Region- 1 currents and the Region- 2 currents are largely under-represented. Comparison of the observed and simulated Birkeland current distributions, which are intimately related to the plasma drifts at the ionosphere, shows that the ionospheric convection pattern in the MHD model and its dependence on the IMF orientation is essentially correct. The Birkeland total currents in the simulations are about a factor of 2 larger than observed during southward IMF. For $B_{z}>0$ the disparity in the total current is reduced and the simulations for purely northward IMF agree with the observations to within 10\%. The disparities in the magnitudes of the Birkeland currents between the observations and the simulation results are a combined effect of the simulation overestimating the ionospheric electric field and of the Iridium fits underestimating the magnetic perturbations. The marginal difference in the latitude resolution of
\end{abstract}

Correspondence to: H. Korth

(haje.korth@jhuapl.edu) the ionospheric grids in the simulations and the observations is shown to have only secondary effect on the magnitudes of the Birkeland currents. The electric potentials in the simulation for southward IMF periods are twice as large as those obtained from measurements of the plasma drift velocities by DMSP, implying that the reconnection rates in the simulation are too large.

Keywords. Ionosphere (Electric fields and currents; Ionosphere-magnetosphere interactions; Modeling and forecasting)

\section{Introduction}

Global magnetohydrodynamic (MHD) models are the most comprehensive numerical tool for studying the coupling of energy and momentum of the solar wind into the Earth's magnetosphere and ionosphere. A particular advantage of global MHD simulations is the ability to provide continuous temporal and spatial coverage of key physical parameters over the entire simulation volume. For this reason, MHD simulations have become one of the principal tools for studying space weather events such as the interaction of the Earth's magnetosphere with coronal mass ejections (CMEs) (Ridley et al., 2002) as well as magnetic storms (Slinker et al., 1998; Goodrich et al., 1998) and substorms (Lyon et al., 1998; Lopez et al., 1998; Wiltberger et al., 2000). Since the simulation results are frequently used to interpret physical processes in the magnetosphere-ionosphere system, assessing their accuracy by comparison with observations is an important task. A number of such studies have been carried out in the past using space-based (Frank et al., 1995; Raeder et al., 1997) and ground-based observations (Ridley et al., 2001), or a combination thereof (Fedder et al., 1998; Slinker et al., 1999). However, interpreting the discrepancies between model and observations is not straightforward because the observational

Published by Copernicus Publications on behalf of the European Geosciences Union. 
data are often sparse compared to the size of the simulation volume and the number of physical quantities evaluated therein.

Recently, Korth et al. (2004), hereinafter P1, compared two-dimensional distributions of Birkeland currents derived from magnetic field observations by the constellation of Iridium satellites with simulation results of the Lyon-FedderMobarry (LFM) MHD model (Fedder and Lyon, 1995; Fedder et al., 1995a; Mobarry et al., 1996) for two intervals where the orientation of the interplanetary magnetic field (IMF) was southward and stable for prolonged periods of time but for which the sign of the IMF $B_{y}$ was opposite. The IMF during these intervals was stable in direction to within $\pm 25^{\circ}$ of the average direction for at least ten hours. The simulations were driven by solar wind (McComas et al., 1998) and IMF (Smith et al., 1998) data from the Advanced Composition Explorer (ACE), located at the first Lagrangian point, L1, starting at least twelve hours prior to the beginning of the respective event period. A detailed comparison of the distributions and magnitudes of the field-aligned currents of the simulation with Iridium observations of the Northern Hemisphere Birkeland currents was then carried out for the last three hours of each event using two different resolutions of the simulation grid, $3^{\circ}$ and $6^{\circ}$ at the ionosphere, and two different planetocentric distances for the simulation inner boundary, $2 R_{E}$ and $3 R_{E}$. The grid resolution was found to be a key factor determining both distributions of the Birkeland currents and their magnitudes. The higher resolution reproduced the location of dayside Region-1 current system within the resolution of the simulation. On the nightside, the simulated Region 1 showed large disparities with respect to the observations caused by spurious gradients in the distribution of the Hall conductance in the LFM. The magnitudes of the simulated currents were about twice as large as observed. The difference was attributed in part to the coarse sampling of the magnetic perturbations in latitude (along the tracks) by the Iridium constellation, which limits the data point density and thus the latitude scales that can be resolved by the spherical harmonic analysis. Because the magnetospheric drift physics is not included in the MHD model, the Region-2 current system was largely under-represented in the simulations, implying that the path of current closure in the simulations departs from that in the natural system. The study presented in P1 provided evidence for an increased flow of electric current across the polar cap and current closure via the simulation inner boundary.

The Iridium constellation of low-altitude satellites offers an opportunity to estimate the low-altitude Birkeland currents without relying on estimates of auroral conductivities. The Iridium satellites provide measurements of the magnetic perturbation signatures due to auroral zone Birkeland currents, allowing distributions of the field-aligned current density to be determined globally. The Iridium network consists of more than 70 satellites distributed over six orbit planes in $780-\mathrm{km}$ altitude circular polar orbits. The satellites are equipped with 30-nT resolution engineering magnetometers, the data of which are routinely used to derive global maps of Birkeland currents (Anderson et al., 2000, 2002; Waters et al., 2001). The sampling period of the Iridium engineering magnetometer data is on average about $200 \mathrm{~s}$ on an individual satellite. Using one hour of data, the global distribution of the large-scale Birkeland currents can be derived with a resolution in latitude of about $4^{\circ}$ and a local-time resolution determined by the $30^{\circ}$ longitude spacing between orbit planes (Anderson et al., 2000; Waters et al., 2004; P1). These twodimensional observations map to a large fraction of the simulation volume and allow for assessments of MHD simulation results of the current density mapped to low altitudes that are more direct than those obtained from comparison with auroral emissions (see P1).

Both observed and simulated Birkeland current distributions in P1 show distinct differences with respect to the sign of IMF $B_{y}$. For positive IMF $B_{y}$, a downward current is found at lower latitudes near local noon with a poleward upward current. The sense in direction of these currents is opposite for negative IMF $B_{y}$. The distribution of field-aligned currents is closely related to the convection of plasma in the ionosphere (Reiff and Burch, 1985), and the Iridium Birkeland current observations may be used to validate the ionospheric convection pattern and its dependence on the IMF orientation. In this paper we expand on our previous work and perform detailed comparisons of the observed Birkeland current distributions with simulation results for geomagnetically disturbed conditions. Comparing intervals spanning a wide range of IMF orientation we evaluate (1) whether the MHD model correctly reflects the basic topology of the ionospheric convection; (2) whether the storm-time expansion of the auroral oval is adequately captured in the MHD model; (3) whether the convection in the MHD model is still too strong and, if so, whether the overestimate of the convection depends on the IMF orientation; and (4) whether the conditions at the ionosphere are still affecting the simulation results. We present Birkeland current observations and simulation results for two magnetic clouds, 17-19 August 2003 and 19-21 March 2001, which are both moderate magnetic storms with minimum $D_{s t}$ of $-168 \mathrm{nT}$ and $-149 \mathrm{nT}$, respectively. The computation of the Birkeland currents from Iridium magnetic field observations and the MHD model are described in Sect. 2. The solar wind observations and the comparison of the observed Birkeland currents with the MHD simulation results are presented in Sect. 3. The results and implications are discussed in Sect. 4, and the findings are summarized in Sect. 5.

\section{Data analysis and event simulation}

We reduce the Iridium magnetometer data as described by Anderson et al. (2000) and derive the low-altitude Birkeland currents using the spherical harmonic fitting technique 
developed by Waters et al. (2001). The Birkeland currents are thereby approximated as radial currents, given in a spherical coordinate system with the intersection of the satellite orbit tracks at its origin as

$j_{\|} \approx j_{\mathrm{r}}=\frac{1}{\mu_{0}} \frac{1}{r \sin \theta}\left(\frac{\partial}{\partial \theta}\left(\sin \theta b_{\phi}\right)-\frac{\partial}{\partial \phi} b_{\theta}\right)$,

where $b_{\theta}$ and $b_{\phi}$ represent the latitudinal and longitudinal components of the magnetic deviation from the main-field, respectively, $\theta$ is the polar angle, and $\phi$ is the azimuth angle. The fit results are transformed to AACGM coordinates (Baker and Wing, 1989) for comparison with the simulations. The uncertainty of the field-aligned current density is given by

$\delta j_{\|} \approx \delta j_{\mathrm{r}}=\frac{1}{\mu_{0} r} \frac{\delta b_{\mathrm{c}}}{\sqrt{n_{\mathrm{b}}}} \sqrt{\frac{2 l^{2}}{\theta_{\max }^{2}}+\frac{1}{\tan ^{2} \theta}+\frac{2}{\sin ^{2} \theta(\delta \phi)^{2}}}$,

where $\delta b_{\mathrm{c}}$ is the standard deviation of the residual difference between the observations and the spherical harmonic fit, $n_{\mathrm{b}}$ is the average number of samples in a half wavelength of the highest latitude order term in the fit, $\theta_{\max }$ is the maximum polar angle used for the fit, $\delta \phi$ the the azimuth spacing between orbit tracks $(\pi / 6)$, and $l$ is the latitude order of the fit (P1). To acquire a data point density along the satellite tracks that is sufficient to derive the Birkeland currents at a latitude resolution of $4^{\circ}$, data accumulation of at least one hour is required.

To minimize time-aliasing due to dynamic variations during the Iridium data accumulation period, the IMF forcing of the magnetosphere needs to be consistent for the entire sample duration. The most dominant driver determining the distribution of the Birkeland currents is the IMF clock angle $\alpha=\arctan \left(B_{y} / B_{z}\right)$, i.e. the angle of the IMF vector in the GSM $y-z$ plane with respect to the northward $(+z)$ direction. The analysis of Birkeland currents is therefore limited to periods during which the IMF orientation varies only slowly in time. For the two events presented here, 17-19 August 2003 and 19-21 March 2001, the clock angle varied on average by $\sim 10^{\circ}$ per hour. Visual inspection of the magnetic perturbation vectors showed that this IMF rotation rate is sufficiently low to yield Birkeland current signatures at the ionosphere that are coherent for periods up to three hours in length. Therefore, we use data accumulation intervals of one to three hours and survey the vector magnetic perturbations for coherency in order to ensure that the Birkeland current configuration at the ionosphere does not experience significant changes during this period.

The simulation we use is the Lyon-Fedder-Mobarry MHD simulation model for the coupled solar wind-magnetosphereionosphere system (Lyon et al., 2004). The model solves the ideal MHD equations inside a cylindrical volume, whose axis of symmetry is aligned with the $\mathrm{x}$-axis of the solar magnetic (SM) coordinate system. Its dimensions extend from $x=+30 R_{E}$ to $x=-300 R_{E}$ in length with a radius of $60 R_{E}$ in the $y$-z-plane of the solar magnetic coordinate system. The inner simulation boundary is located at $2 R_{E}$ radial distance from the Earth's center. As addressed in P1, the simulation inner boundary must be low enough to ensure that the source regions of the Birkeland currents are contained within the simulation volume. Since Birkeland currents often expand well equatorward of $60^{\circ}$ MLAT, their sources are not included in many typical MHD simulations which presently place the simulation inner boundary at $3 R_{E}$. The computational mesh features a distorted spherical coordinate system, designed to maximize the resolution at the magnetopause, the ionosphere, and the geomagnetic tail, with coarser resolution far from the Earth in the solar wind and the magnetosheath. The spacing of the numerical grid points in the simulation has a significant impact on the distribution of field-aligned currents and their magnitudes, and higher resolutions yield better qualitative and quantitative comparisons with observations (see P1). The grid resolution of the simulation results presented here corresponds to a latitude resolution of $\sim 3^{\circ}$ in the ionosphere at $70^{\circ}$ MLAT, denoted "high resolution" in P1.

The simulations are driven by solar wind and IMF data starting at least twelve hours prior to the arrival of the magnetic cloud at Earth. We use ACE data (McComas et al., 1998; Smith et al., 1998) for the 17-19 August 2003 interval and WIND data (Ogilvie et al., 1995; Lepping et al., 1995) for the 19-21 March 2001 period, during which the ACE plasma data is not continuously available. The solar wind conditions are imposed as outer boundary conditions on the upstream and side boundaries, while an outflow conditions exists at the downstream boundary. The inner boundary conditions are determined from a two-dimensional ionospheric simulation, solving the potential equation

$\nabla_{\perp} \cdot \Sigma \cdot \nabla_{\perp} \phi=j_{\|} \sin \delta$

to obtain the electric potential, $\phi$, from the field-aligned Birkeland currents, a model for the anisotropic conductance tensor, $\boldsymbol{\Sigma}$, and the magnetic field dip angle, $\delta$. The conductance model includes contributions of both solar EUV illumination and auroral particle precipitation. For a detailed discussion of LFM conductance model please refer to the description by Fedder et al. (1995b), which is also summarized in $\mathrm{P} 1$.

\section{Event descriptions}

\subsection{7-19 August 2003}

The solar wind conditions at the ACE location for the 1719 August 2003 magnetic cloud are shown in Fig. 1. From top to bottom the panels show the IMF $B_{x}, B_{y}$ and $B_{z}$ components in GSM coordinates; the IMF magnitude, $B_{t}$; the IMF clock angle, $\alpha$; the proton number density, $n_{\mathrm{p}}$; the proton bulk speed, $v_{\mathrm{p}}$; and the proton dynamic pressure, $p_{\text {dyn }}$. 


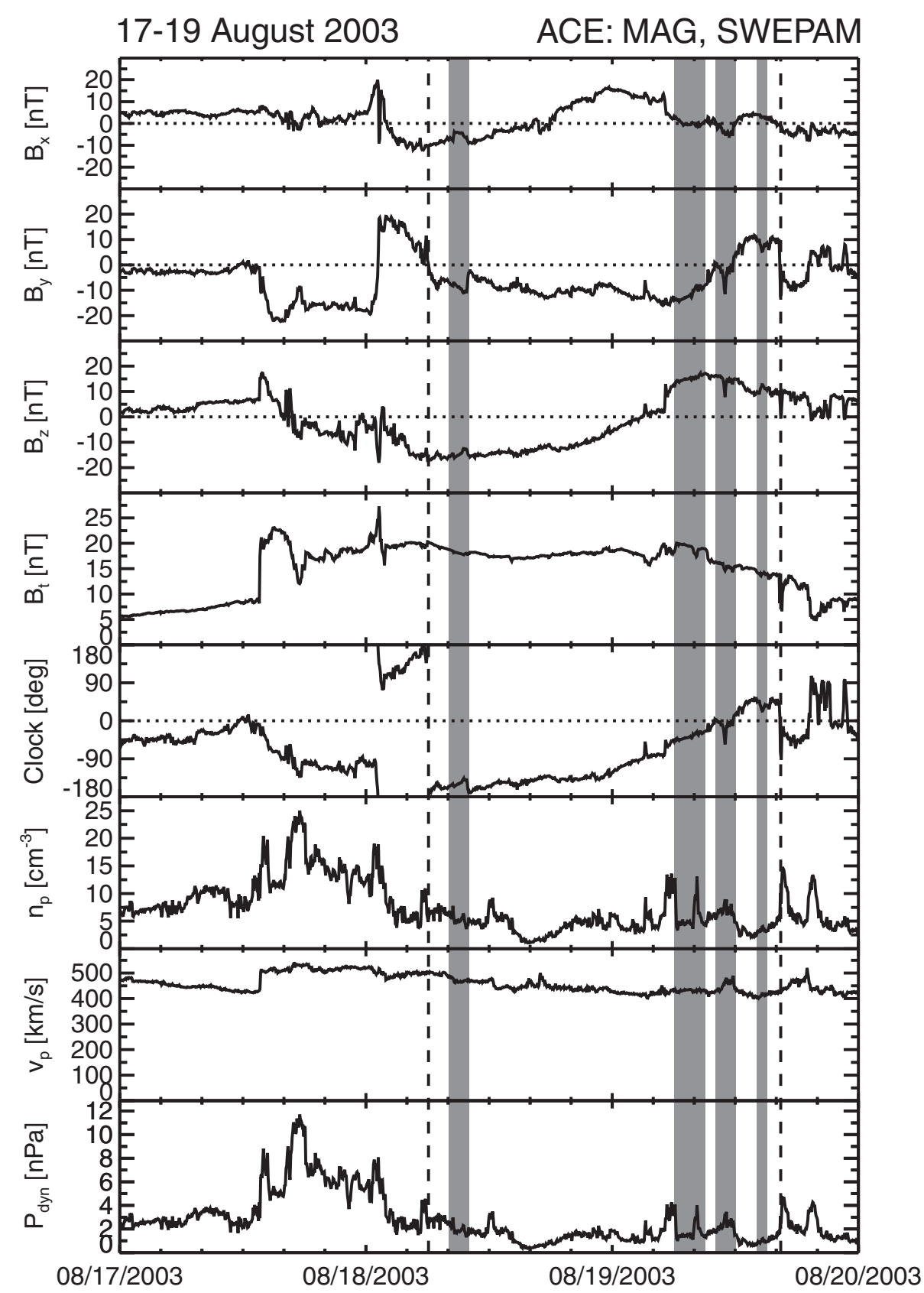

Fig. 1. Solar wind conditions for 17-19 August 2003 as observed by ACE. The interval of the study is marked by vertical dashed lines.

The arrival of the sheath of the CME at ACE on $17 \mathrm{Au}$ gust 2003 at 13:45 UT is associated with sudden increases of the IMF magnitude from $7 \mathrm{nT}$ to $20 \mathrm{nT}$ and the solar wind dynamic pressure from $2 \mathrm{nPa}$ to $8 \mathrm{nPa}$, largely caused by a threefold solar wind density enhancement. The hours following the shock arrival are characterized by large fluctuations of the IMF and the solar wind plasma parameters. By $18 \mathrm{Au}-$ gust 2003, 06:00 UT, the solar wind pressure reverts to $2 \mathrm{nPa}$, marking the beginning of the actual cloud passage, which is characterized by a slow rotation of the IMF from southward to northward with negative IMF $B_{y}$. The IMF $B_{y}$ then turns positive while the IMF $B_{z}$ maintains northward orientation. The magnetic cloud event ends at 16:30 UT on 19 August 2003, when the IMF abruptly changes direction and becomes variable again. We focus here on the interval of slow rotation of the IMF demarcated by the two vertical dashed lines in Fig. 1, where the rate of change of the IMF clock angle is on average $10.8^{\circ} / \mathrm{h}$ (median $8.2^{\circ} / \mathrm{h}$ ). The average magnitudes and standard deviations of the IMF and solar wind speed during this interval are $18 \pm 2 \mathrm{nT}$ and $460 \pm 40 \mathrm{~km} / \mathrm{s}$, 
respectively. The time lag for the arrival of the solar wind conditions at Earth, obtained from simple advection from the ACE location to Earth, is $55 \mathrm{~min}$.

The Iridium Birkeland current distributions are shown together with the LFM simulation results in Fig. 2 for four different intervals within the event period. The observed Birkeland current densities are shown in the left hand panel with upward and downward currents shown in red and blue, respectively. Regions where the current densities are lower in magnitude than twice the standard deviation are shaded gray in the figure. The right hand figure in each panel shows the LFM simulation results, where the green and black solid contours represent the two standard deviation confidence level of the observed upward and downward field-aligned currents, respectively. Note that the color bar for the simulation results spans a factor of two greater range than the observed currents. The intervals shown in Fig. 2 have been selected to demonstrate the dependence of the Birkeland current distributions on the IMF orientation. Periods that resemble distributions similar to ones shown in Fig. 2 have been omitted.

Figure 2a shows the distribution of Birkeland currents on 18 August 2003 from 08:00-10:00 UT near the beginning of the event period. The IMF clock angle averages $-150^{\circ}$ during this time, and the observations clearly show the largescale Region-1 and Region-2 current systems, which are commonly observed by Iridium during southward IMF and documented statistically by Iijima and Potemra (1978). Furthermore, the observed distribution of the Birkeland current densities shows an asymmetry with respect to the noonmidnight meridian, which is consistent with the description by Erlandson et al. (1988) for negative IMF $B_{y}$. To compare the total Region-1/2 currents in the simulation with their observed magnitudes, we integrate the current densities above the $2 \sigma$ confidence level over the respective region. The observed Region- 1 total currents is $3.8 \mathrm{MA}$ on the dawnside and 3.3 MA on the duskside, and the total Region2 currents are 3.5 MA and 2.8 MA at dawn and dusk, respectively. The LFM simulation results clearly show the dayside Region-1 current system, the distribution of which compares generally well with the observations, although the axis of symmetry in the simulation is closer to noon than is observed. The disparity between the observed and simulated field-aligned currents on the nightside is due to spurious gradients in the ionospheric conductances generated by the precipitation model implemented in the LFM, which have been previously discussed in P1. In comparison with the observations, the Region- 1 currents of the simulation exhibit larger magnitudes, 7.4 MA at dawn and 8.0 MA at dusk. The Region-2 Birkeland currents of the simulation in Fig. 2a are 0.6 MA on the dawnside and 0.7 MA at dusk, on average a factor of five lower than the Iridium results. Additional fieldaligned currents are evident in the simulations results on the dayside near $50^{\circ}$ MLAT, just poleward of the magnetic latitude at which the $2 R_{E}$ simulation inner boundary maps to the ionosphere. These boundary currents have been previously
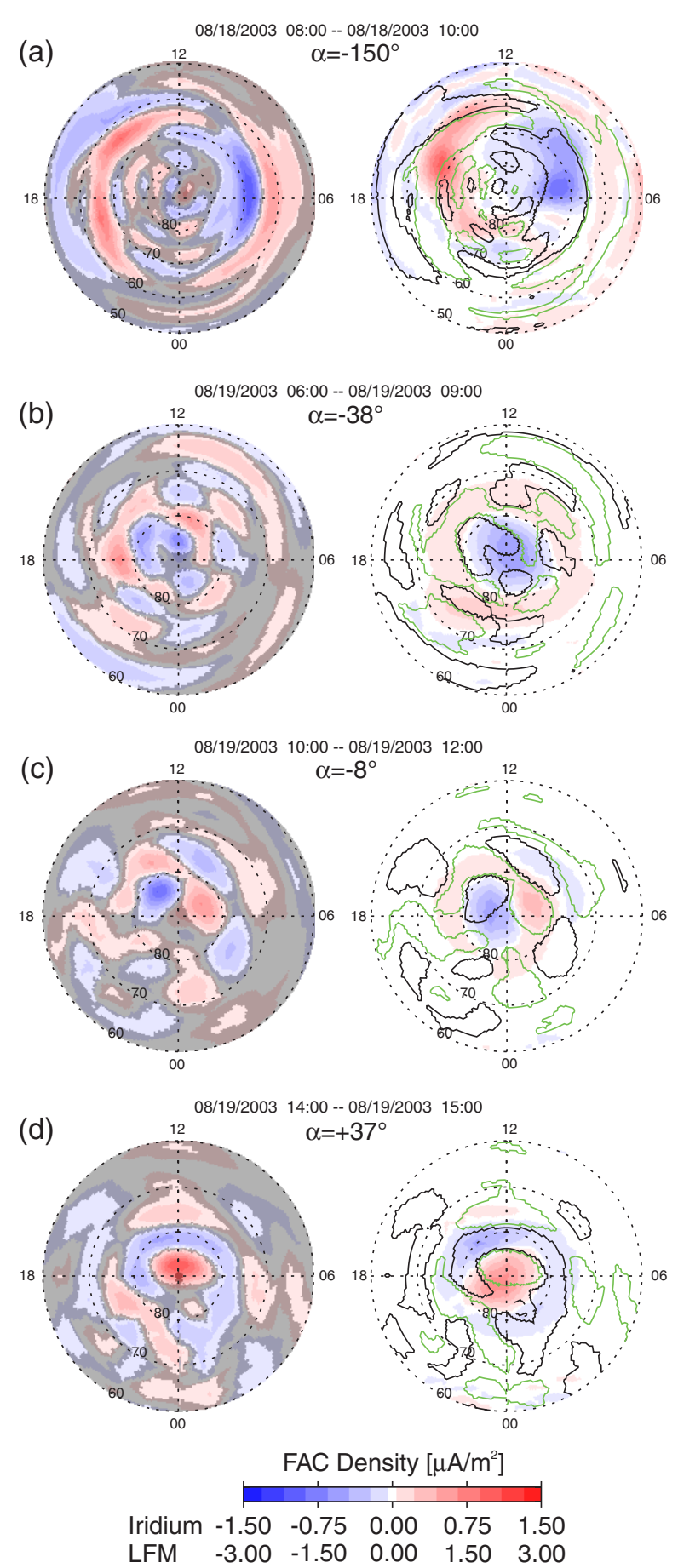

Fig. 2. Comparison of Birkeland current distributions (left panels) observed during the 17-19 August 2003 magnetic cloud with LFM simulations results (right panels). Downward and upward currents are represented by the colors blue and red, respectively. 
identified to compensate for the under-representation of Region 2 and provide a means for closure of the Region-1 currents in the simulation (P1). It should be noted that the IMF clock angle during this interval is similar to the one of the 31 March 2000 event discussed in P1, and the comparison of the observations and the simulation results for that event is qualitatively similar to the characteristics described above.

To evaluate the relative location in latitude of the observed and simulated Birkeland currents, we calculate the differential total current, i.e. the total current, $I$, per unit polar angle, $\theta$,

$$
\delta I\left[\mathrm{MA} /{ }^{\circ}\right]=\frac{d I}{d \theta}=\int_{\phi_{1}}^{\phi_{2}} j_{\|} \mathrm{d} s,
$$

for a two-hour range in magnetic local time from $\phi_{1}$ to $\phi_{2}$ at dawn, 06:00-08:00 MLT, and at dusk, 16:00-18:00 MLT. In Eq. (4), all current densities within the respective local time range are integrated, independent of their magnitude with respect to the $2 \sigma$ uncertainty estimate. Figure 3 shows the observed differential total current (solid line) on $18 \mathrm{Au}$ gust 2003, 08:00-10:00 UT, as a function of magnetic latitude together with the simulation results (dotted line), where positive values represent upward currents. On the duskside, the observations show the maximum $\delta I$ of Region 1 at $65^{\circ}$ MLAT and a dawnside peak at $69^{\circ}$ MLAT. In comparison, the simulation places the peaks of Region 1 at $68^{\circ}$ and $72^{\circ}$ at dusk and dawn, respectively, poleward with respect to the observations by $3^{\circ}$ at both dawn and dusk. This is similar to the poleward displacement by $5^{\circ}$ found in $\mathrm{P} 1$.

After an extended interval of southward IMF, the IMF turned northward at 03:00 UT on 19 August 2003, and Fig. 2b-d (left panels) shows the Birkeland current observations for the subsequent rotation of the IMF from dawnward to duskward for $B_{y}<0, B_{y} \approx 0$, and $B_{y}>0$, respectively. The mean IMF clock angles for these periods are $-38^{\circ},-8^{\circ}$, and $+37^{\circ}$, respectively. During northward IMF the broadly expanded Region-1/2 current system seen in Fig. 2a no longer exists. Instead, the Birkeland currents are organized at high latitudes and their distributions are sensitive to the direction of the IMF $B_{y}$. For $B_{y}<0$ (Fig. 2b), a downward fieldaligned current is located in the region near the magnetic pole with an equatorward upward current. On the other hand, an upward field-aligned current is observed near the magnetic pole for $B_{y}>0$ and the direction of the complementary current at lower latitudes is downward (Fig. 2d). The observations in Fig. 2c show the high-latitude current system that is commonly observed during intervals of strongly northward IMF (e.g. Korth et al., 2005) and which has previously been termed NBZ currents (Iijima et al., 1984; Zanetti et al., 1984; Iijima and Shibaji, 1987). The above observations at high latitudes agree qualitatively with the predictions by Reiff and Burch (1985). Additional field-aligned currents are observed at lower latitudes. However, their occurrence is not system- atic and their distribution varies considerably in time, indicating that these currents are either transient or an artifact.

For all three intervals, the simulation results in Fig. 2b$\mathrm{d}$ (right panels) reproduce the observed dependence of the high-latitude Birkeland current distribution on the IMF orientation remarkably well. The Birkeland currents in the simulation are located predominantly at latitudes poleward of $70^{\circ}$ MLAT and do not show the smaller-scale current features observed at lower latitudes. To compare the observations and the simulation results during northward IMF quantitatively, we calculate the average magnitude of the upward and downward field-aligned currents poleward of $70^{\circ}$ MLAT. The observed total currents in Fig. 2b, c, and d amount to 1.40 MA, 1.33 MA, and 1.55 MA, respectively, while the simulation yields 2.34 MA, 1.41 MA, and 2.02 MA for these intervals. Comparison of the two sets of total currents shows that the total currents for purely northward IMF (Fig. 2c) agree to within $10 \%$, whereas the simulation results in Fig. $2 \mathrm{~b}$ and $\mathrm{d}$ exceed the observations on average by $\sim 50 \%$.

\subsection{9-21 March 2001}

For the period of 19-21 March 2001 the solar wind plasma data, which is used together with the IMF observations to drive the MHD simulation, is not continuously available from ACE. Therefore, we use solar wind and IMF observations by the WIND spacecraft for this event. The approximate location of WIND during the above interval in GSE coordinates was $X=-15 R_{E}, Y=-200 R_{E}$, and $Z=6 R_{E}$. The IMF and solar wind parameters observed by the Magnetic Field Investigation (MFI) instrument (Lepping et al., 1995) and the Solar Wind Experiment (SWE) (Ogilvie et al., 1995) for 19-21 March 2001 are shown in Fig. 4 in the same format used in Fig. 1. Strong magnetic field fluctuations associated with the sheath of the CME are evident in the WIND observations beginning 10:00 UT on 19 March 2001. On 21 March 2001, 22:00 UT, the large variability ceases and the IMF evolves into a period of slow rotation from the dawnward to the duskward direction with $B_{z}<0$ at a rate of on average $7.0^{\circ} / \mathrm{h}$ (median 5.4\% $/ \mathrm{h}$ ) until 21 March 2001, 22:00 UT. During this interval, the IMF magnitude averages $14.5 \pm 2.9 \mathrm{nT}$ and the mean solar wind speed is $375 \pm 45 \mathrm{~km} / \mathrm{s}$. Due to the proximity of WIND to the dayside magnetopause, the advective delay is ignored since it is only a few minutes.

Representative distributions of the observed and simulated Birkeland currents are shown in Fig. 5. Figure 5a shows the current distribution for 12:00-15:00 UT on 20 March 2001, where the IMF is oriented southward and duskward with a mean direction corresponding to a clock angle of $166^{\circ}$. The observed Region-1/2 Birkeland current system (left panel) shows an asymmetry with respect to the noonmidnight meridian, typical for IMF $B_{y}>0$ (Erlandson et al., 1988), which was also observed during an interval on 23 November 1999 discussed in P1. This asymmetry is not evident in the simulations results (right panel). For quantitative 


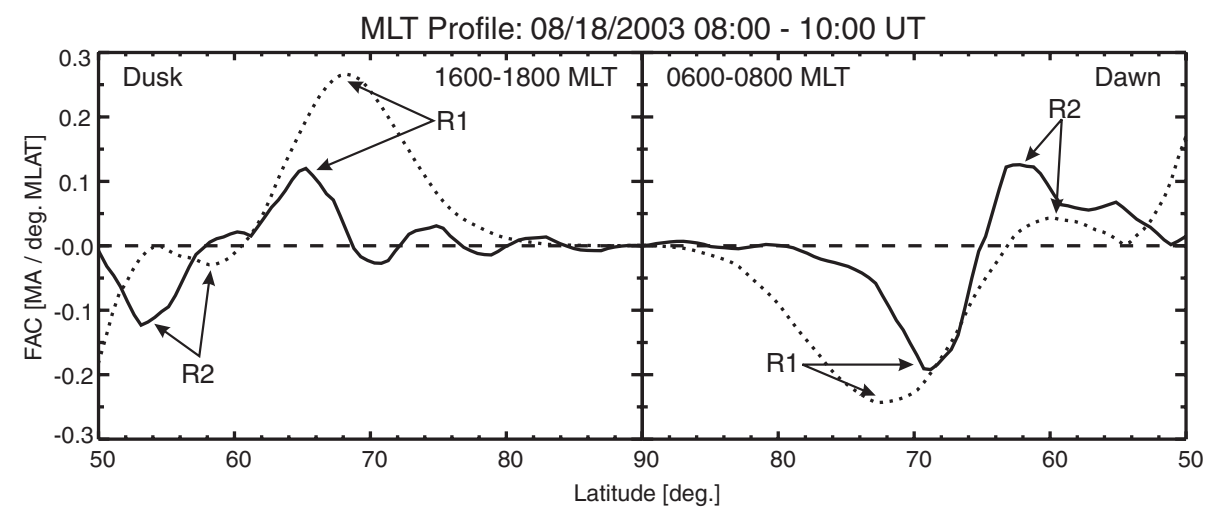

Fig. 3. Differential total field-aligned currents from Iridium (solid line) and LFM simulation (dotted line) at dawn and dusk for 18 August 2003, 08:00-10:00 UT.

comparison, we integrate the observed Birkeland currents above $2 \sigma$. On the dawnside, the observed Region- 1 current totals 3.6 MA, and its counterpart on the duskside amounts to 3.7 MA. The Region- 2 currents integrate to $2.2 \mathrm{MA}$ at both dawn and dusk. The magnitudes of the Region-1 currents in the simulation are 5.7 MA at dawn and 5.9 MA at dusk, about $50 \%$ larger than observed, while the simulated Region-2 total currents of 0.4 MA at both dawn and dusk, less than $20 \%$ of the observed values. The differential total current at dawn and dusk for this interval is presented in Fig. 6 in the same format used in Fig. 3. The figure illustrates a poleward displacement of Region 1 in the simulation (dotted line) with respect to the observations (solid line) of $6^{\circ}$ on average.

Figure 5b shows the Birkeland currents for 12:0014:00 UT on 21 March 2001, where the orientation of the IMF is nearly duskward with a mean clock angle of $88^{\circ}$. The field-aligned current distribution observed during this interval exhibits an upward current with a magnitude of $0.8 \mathrm{MA}$ in the vicinity of the magnetic pole and an equatorward downward current of 1.2 MA stretching from local midnight into the afternoon sector. The observed distribution is generally well reproduced by the simulation, although the current magnitudes of 2.0 MA and 3.2 MA for the upward and downward currents, respectively, exceed the observations by a factor of $\sim 2.5$. The distributions in Fig. 5a and b are qualitatively similar to the ones presented in Fig. 2a and d, demonstrating both the consistency of the Iridium observations and reproducibility of the observed distributions by the MHD model.

\section{Discussion}

\subsection{Birkeland current distributions}

The topology of the field-aligned current distributions shown in Figs. 2 and 5 is intimately tied to the large-scale plasma circulation in the ionosphere and magnetosphere, which in turn is governed by the orientation of the IMF (Burch et al., 1985; Reiff and Burch, 1985). During southward IMF, the ionospheric convection is anti-sunward across the polar cap with a sunward return flow at lower latitudes, forming the two-cell convection pattern commonly observed under these conditions. The plasma circulation within these cells is clockwise (counter-clockwise) at dusk (dawn) and is in the Northern Hemisphere associated with convergent (divergent) electric fields, driving horizontal currents in the ionosphere. In the dusk (dawn) convection cell, upward (downward) field-aligned Region-1 Birkeland currents, are easily identified in the observations shown in Figs. 2a and 5a and their distributions are captured well by the simulation. On the nightside, the Iridium observations and simulation results show larger difference caused by spurious gradients in the Hall conductance distribution at the poleward edge of the auroral oval, which have been discussed in detail in P1. While the dayside Region-1 currents are generally well reproduced by the MHD model, the equatorward expansion of these currents during storm times is not. The profiles of the differential total current for 18 August 2003, 08:00 10:00 UT (Fig. 3), and 20 March 2001, 12:00-15:00 UT (Fig. 6), showed that the Birkeland peak current densities in the simulation are located on average $\sim 5^{\circ}$ poleward of their observed location. We suggest that the magnetosphereionosphere system in the simulation reacts to the significant under-representation of the Region-2 currents by displacing the Region-1 current system poleward in an attempt to minimize the energy dissipation in the ionosphere (Barbosa, 1984).

The anti-parallel merging model (Crooker, 1979) predicts a shift of the reconnection site for negative (positive) IMF $B_{y}$ to the dawnside (duskside) magnetopause in the Northern Hemisphere, leading to growth of the dawn (dusk) lobe convection cell (Reiff and Burch, 1985). The divergent (convergent) electric fields inside the enlarged lobe cell are associated with a downward (upward) field-aligned current near 


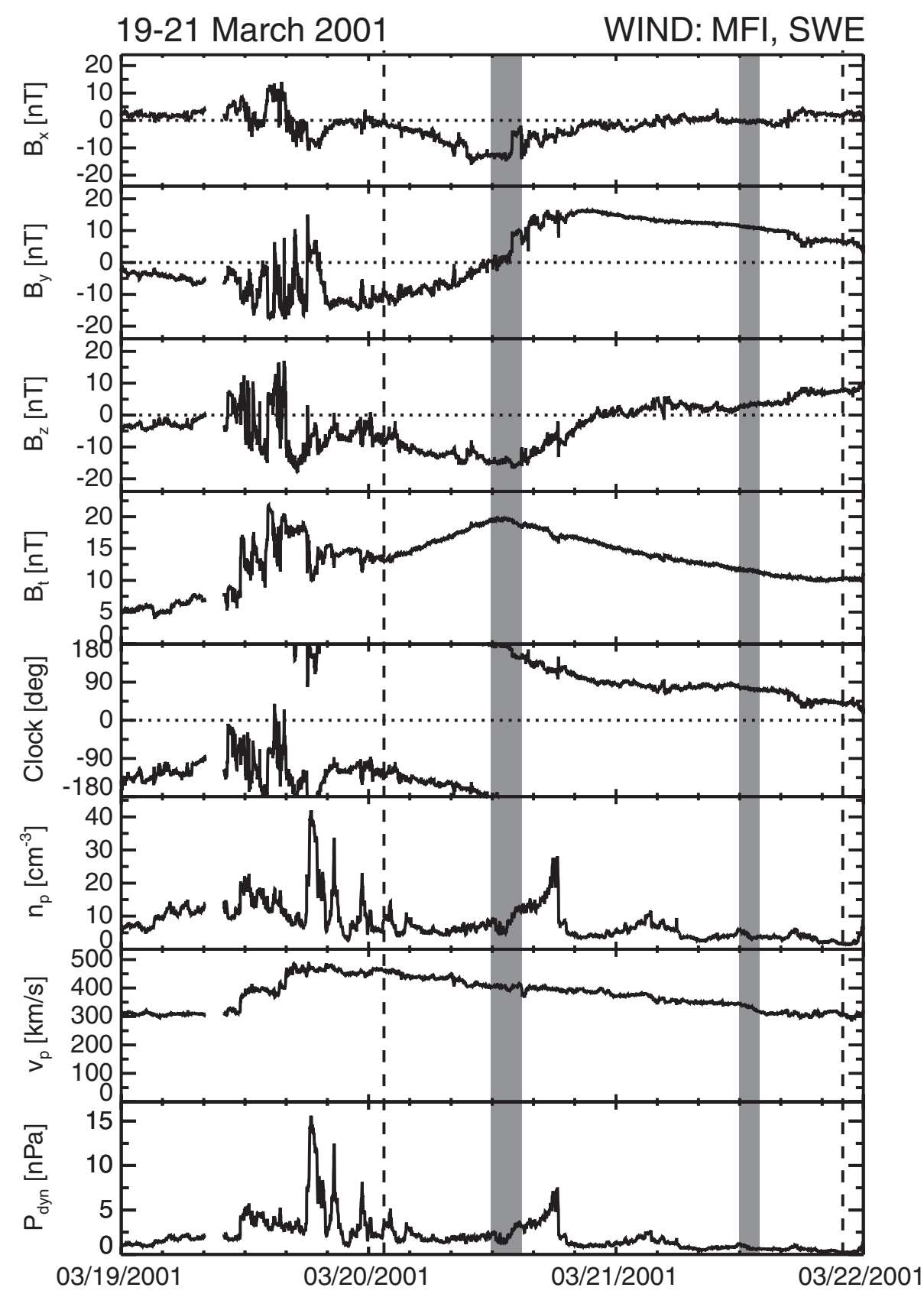

Fig. 4. Solar wind conditions for 19-21 March 2001 as observed by WIND. The interval of the study is marked by vertical dashed lines.

local noon. Equatorward of the lobe cell, the sense of convection reverses leading to the formation of a complementary field-aligned current with opposite polarity (Ohtani et al., 1995). Both the poleward downward and equatorward upward Birkeland currents described above are evident during the interval of negative IMF $B_{y}$ in Fig. 2a, while the polarity of the noon currents is reversed in Fig. 5a, where $B_{y}>0$. The simulation qualitatively reproduces the asymmetry at noon for $B_{y}<0$, while it is not evident in the simulation results for $B_{y}>0$.
During northward IMF conditions, Crooker (1979) predicts a shift of the reconnection site at the subsolar magnetopause to locations poleward of the cusp. The NBZ fieldaligned currents, which are observed by Iridium and matched well by the simulation results for strongly northward IMF (Fig. 2c), are the consequence of the reversed-sense lobe cell convection developing at high latitudes (Russell, 1972; Maezawa, 1976; Reiff and Burch, 1985). These current distributions are also consistent with those obtained from an MHD simulation of a northward-to-duskward turning of the 

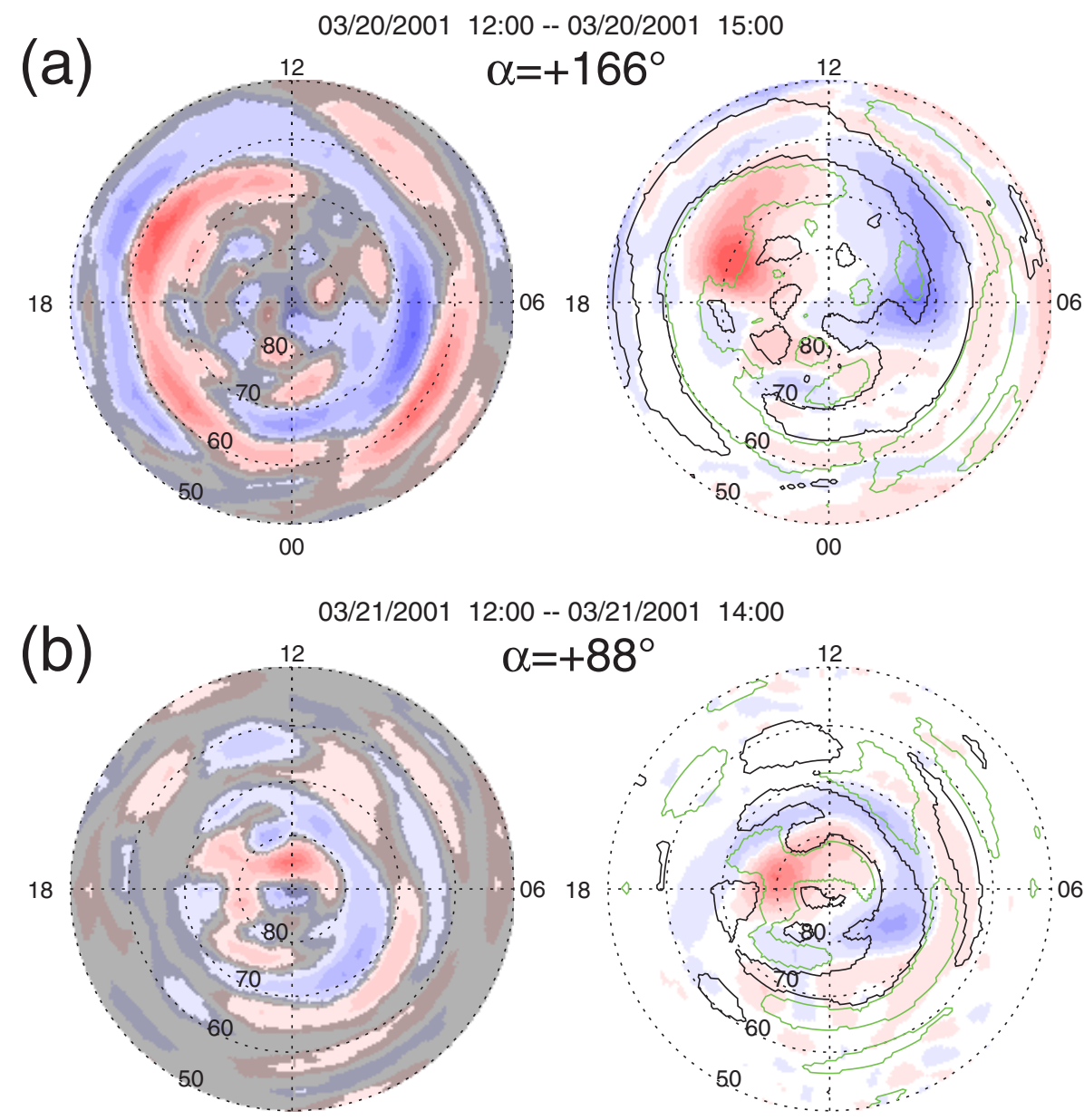

00

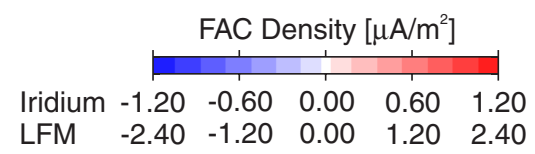

Fig. 5. Comparison of Birkeland current distributions observed during the 19-21 March 2001 magnetic cloud with LFM simulations results. Downward and upward currents are represented by the colors blue and red, respectively.

IMF by Vennerstrøm et al. (2005). The authors show that the NBZ currents map to major portions of the magnetopause and are created by the shear of newly reconnected field lines against the mantle field as they are convected tailward by the solar wind. As the IMF rotates away from the northward direction, the relative size of the lobe cells at dawn and dusk changes. The observed change in the distribution of the Birkeland currents associated with the modification the convection pattern is that the upward (downward) current occupies the region near the magnetic pole for $B_{y}>0\left(B_{y}<0\right)$ (cf. Fig. $2 b$ and d). Both of these features are captured by the MHD model. Furthermore, the Birkeland currents for $B_{y}>0$ agree qualitatively with the distribution in Vennerstrøm et al. (2005) for these conditions. The qualitative agreement of the Iridium observations with the simulation results during northward IMF indicates that for these conditions the change in topology of the convection pattern with varying IMF orientation is adequately reproduced by the LFM.

Although the change in topology of the Birkeland currents with IMF orientation is described above by the paradigm of anti-parallel merging, the current distributions are also consistent with the component-merging theory of reconnection. The MHD equations do not require anti-parallel field-line geometry as a necessary condition for reconnection, and Cowley (1976) suggested that reconnection more generally occurs where the magnetosheath and magnetospheric magnetic fields have oppositely-directed components of equal magnitudes. The component merging model implies that dayside sub-solar reconnection occurs for all IMF orientations having a southward component along an $\mathrm{X}$ line, which is rotated with respect to the equatorial plane for $B_{y} \neq 0$. Observational evidence for component reconnection has been published, 
MLT Profile: 03/20/2001 12:00 - 15:00 UT

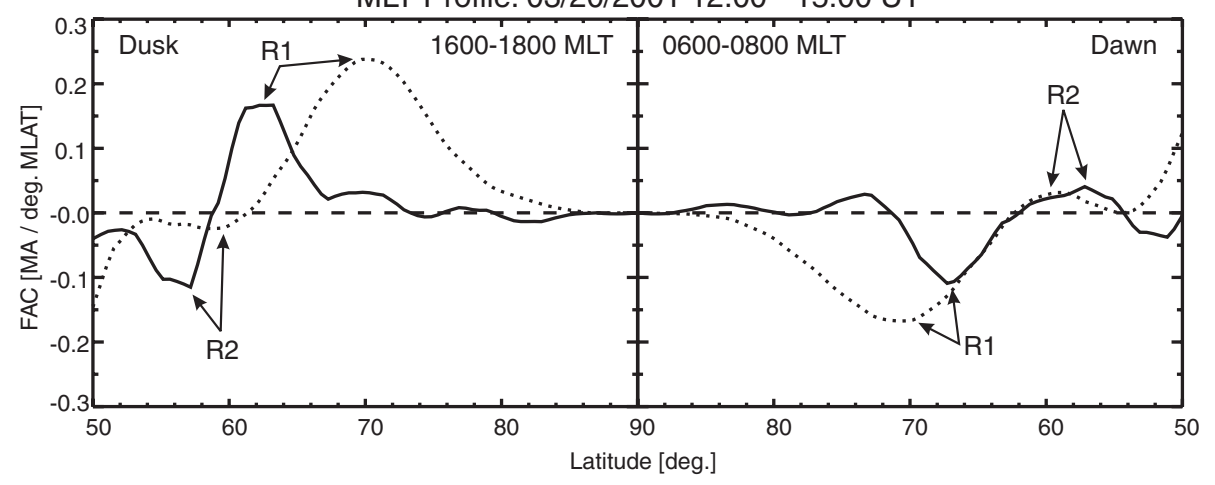

Fig. 6. Differential total field-aligned currents from Iridium (solid line) and LFM simulation (dotted line) at dawn and dusk for 20 March 2001, 12:00-15:00 UT.

among others, by Gosling et al. (1990) and Onsager and Fuselier (1994). Preliminary studies of reconnection using the LFM indicate that for southward IMF both anti-parallel and component merging are evident in the simulation, with anti-parallel reconnection being dominant. For northward IMF, the LFM simulations appear to be more consistent with anti-parallel reconnection. The fact that both anti-parallel and component merging are encountered in the simulation indicates that both types of reconnection occur simultaneously, as suggested by recent theoretical (Moore et al., 2002; Sandholt et al., 2004) and observational studies (Massetti, 2006).

\subsection{Birkeland total current}

The magnitudes of the simulated Birkeland currents for southward IMF conditions (Figs. 2a and 5a) are about twice as large as observed. To compare the simulated and observed total currents over a wider range of the IMF orientation, we calculate the time series of the hemispheric total current in one-hour steps for both events. The integration of the hemispheric total current includes all current densities poleward of $55^{\circ} \mathrm{MLAT}$, and the integration of the Iridium data includes only current densities above the $2 \sigma$ confidence level. Figure 7 shows the average magnitude of the integrated upward and downward hemispheric total current (bottom panel) from the Iridium observations (solid line) and the LFM simulation (dotted line) for 17-19 August 2003 together with the $D_{s t}$ index (top panel). Intervals for which the Birkeland current distributions were presented in Fig. 2 are shaded gray in the figure.

The simulations yield significantly more current than indicated by the observations. The pre-storm magnitudes of the total current in the simulation are in approximate agreement with the observations. At the beginning of the storm main phase a sudden increase in the total current is registered in both observations and simulation results, but the magnitude in the simulation is initially about two times larger than ob- served. The initial difference in the total current diminishes temporarily approximately six hours into the main phase. Nevertheless, the LFM total current exceeds the observed magnitude throughout the early recovery phase on average by $75 \%$ until the pre-storm agreement of the currents is restored as the IMF turns northward at 04:00 UT on 19 August 2003.

For the 19-21 March 2001 event, the observed hemispheric total current, shown in Fig. 8 in the same format as used in Fig. 7, compares similarly well with the simulations on a relative scale, but the latter exceeds the former on average by $78 \%$. Therefore, we conclude that the overestimation of the total current by the simulations is a persistent feature of the comparison of observations and simulation results, which is most prominent during southward IMF. While the simulated currents during northward orientation of the IMF are also larger than observed, the disparity between the observation and the simulation results is reduced under these conditions. That the Birkeland currents are overestimated by the simulation during southward IMF conditions has been previously discussed in $\mathrm{P} 1$, but the fact that the overestimation is a characteristic feature of the model identified for a wide range of solar wind and IMF conditions, is a new result of this study.

It is important to understand that the calculation of the hemispheric total current is not confined to the regions of the large-scale Birkeland currents and thus commonly overestimates these currents. Furthermore, the comparison of the observed and simulated integrated hemispheric total current does not necessarily reflect the level of agreement for specific field-aligned current features. In the observations, a significant fraction of the total current during both southward and northward IMF comes from contributions at lower latitudes, where the currents in the simulation are often underestimated. Therefore, it is possible for disparities to exist at higher latitudes even though the total current in the simulation is in good agreement with the observations. For 


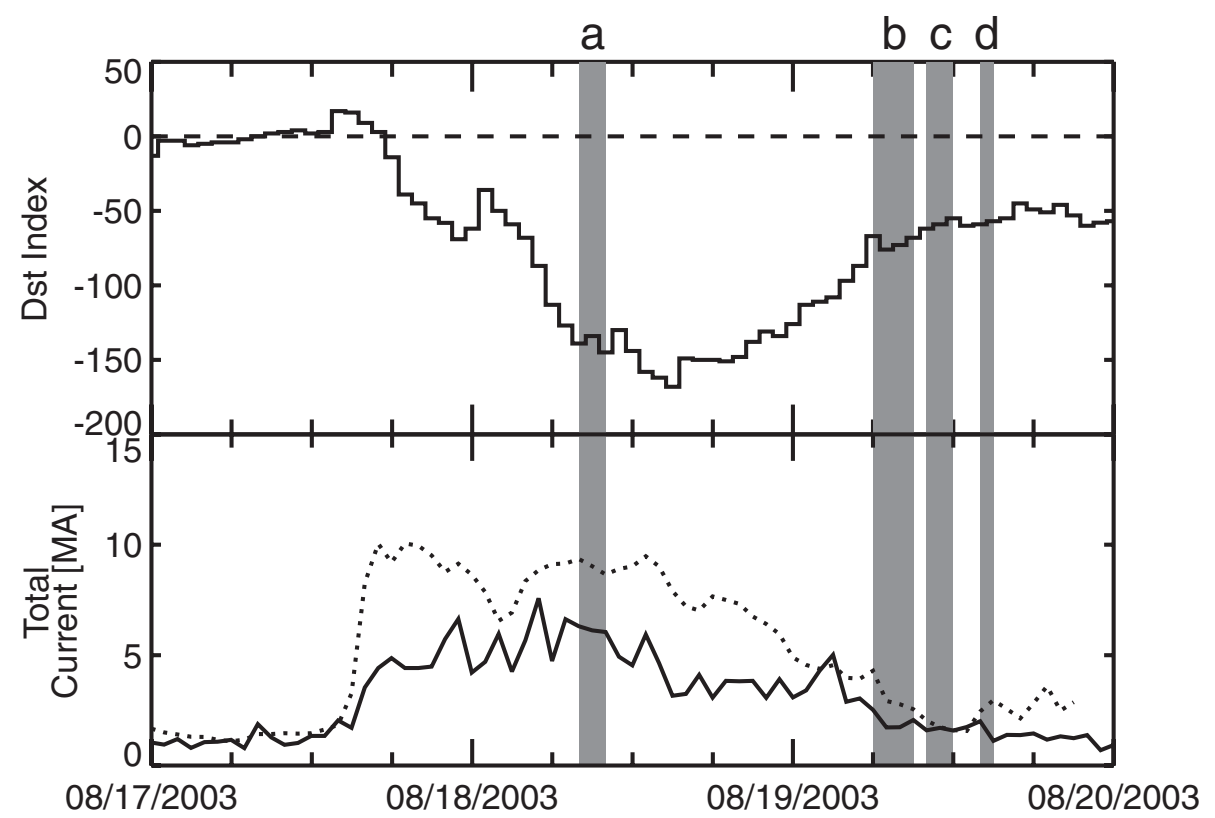

Fig. 7. $D_{s t}$ Index and hemispheric total currents from Iridium (solid line) and LFM (dotted line) for the 17-19 August 2003 magnetic cloud. The intervals shown in Fig. 2 are gray shaded.

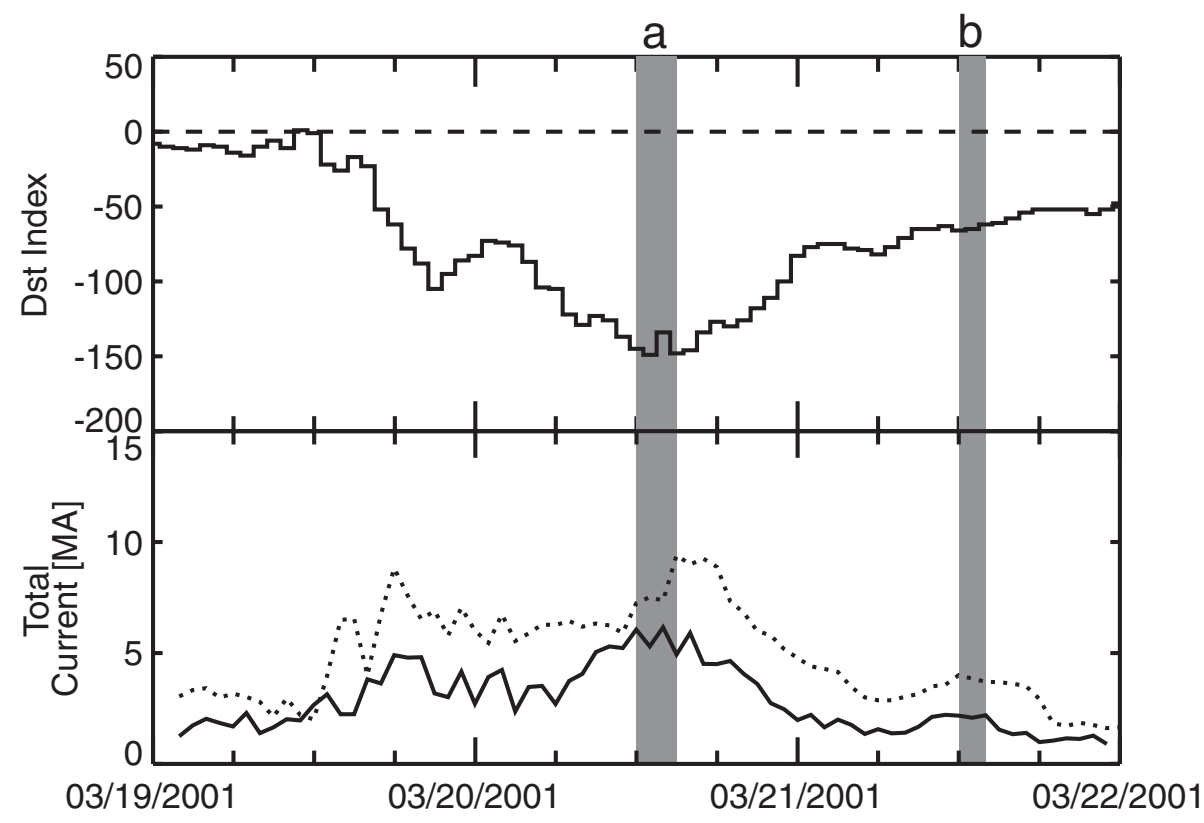

Fig. 8. $D_{s t}$ Index and total currents from Iridium (solid line) and LFM (dotted line) for the 19-21 March 2001 magnetic cloud. The intervals shown in Fig. 5 are gray shaded.

example, during the interval from 12:00-15:00 UT on 20 March 2001 the observed hemispheric total current agrees with the simulation results to within about $20 \%$, yet the magnitude of the Region- 1 currents in the simulation are approximately 60\% larger than observed (cf. Sect. 3 and Fig. 5a).

\subsection{Comparison with DMSP observations}

The estimates for the observed hemispheric total current are low due to the smoothing of the magnetic perturbations by the spherical harmonic fit and this contribution to the 
08/18/2003 0800-1000 UT
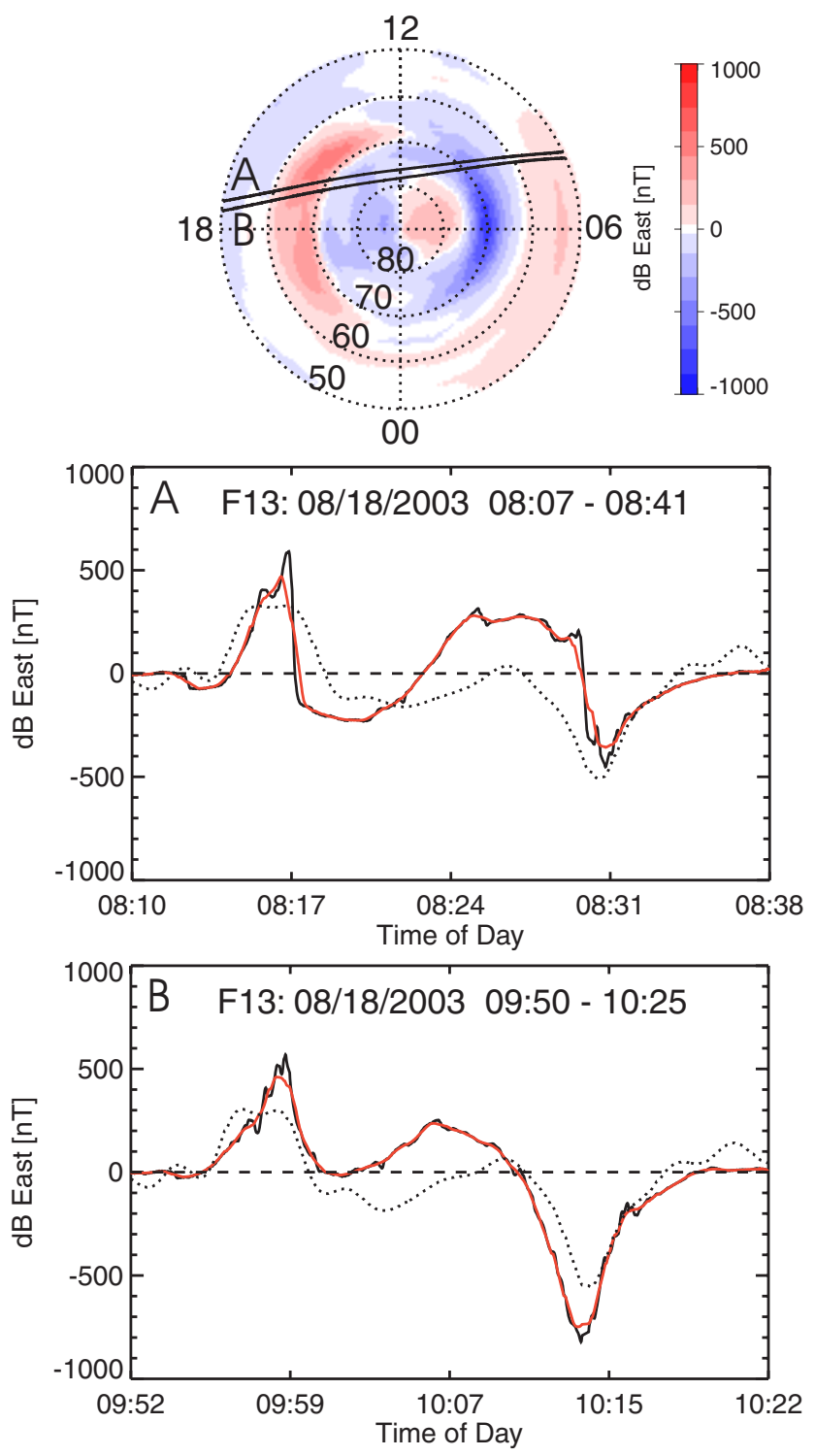

Fig. 9. Comparison of the fitted magnetic perturbations from Iridium (dotted line) with DMSP F13 observation (solid black line) during the interval 18 August 2003, 08:00-10:00 UT. The red solid line shows the $50 \mathrm{~s}$ boxcar average of the DMSP F13 data.

difference vis-à-vis the simulations results needs to be quantified for these cases. While the Iridium inversion technique reliably locates the large-scale Birkeland currents, previous comparisons with data from Ørsted and DMSP have confirmed that the peak magnetic perturbations are often low. Figures 9 and 10 compare the eastward magnetic perturbations observed by DMSP F13 (solid black line) with the fitted eastward magnetic perturbations from Iridium, evaluated along the F13 orbit (dotted lines) for 18 August 2003, 08:0010:00 UT, and 20 March 2001, 12:00-15:00 UT, respectively.
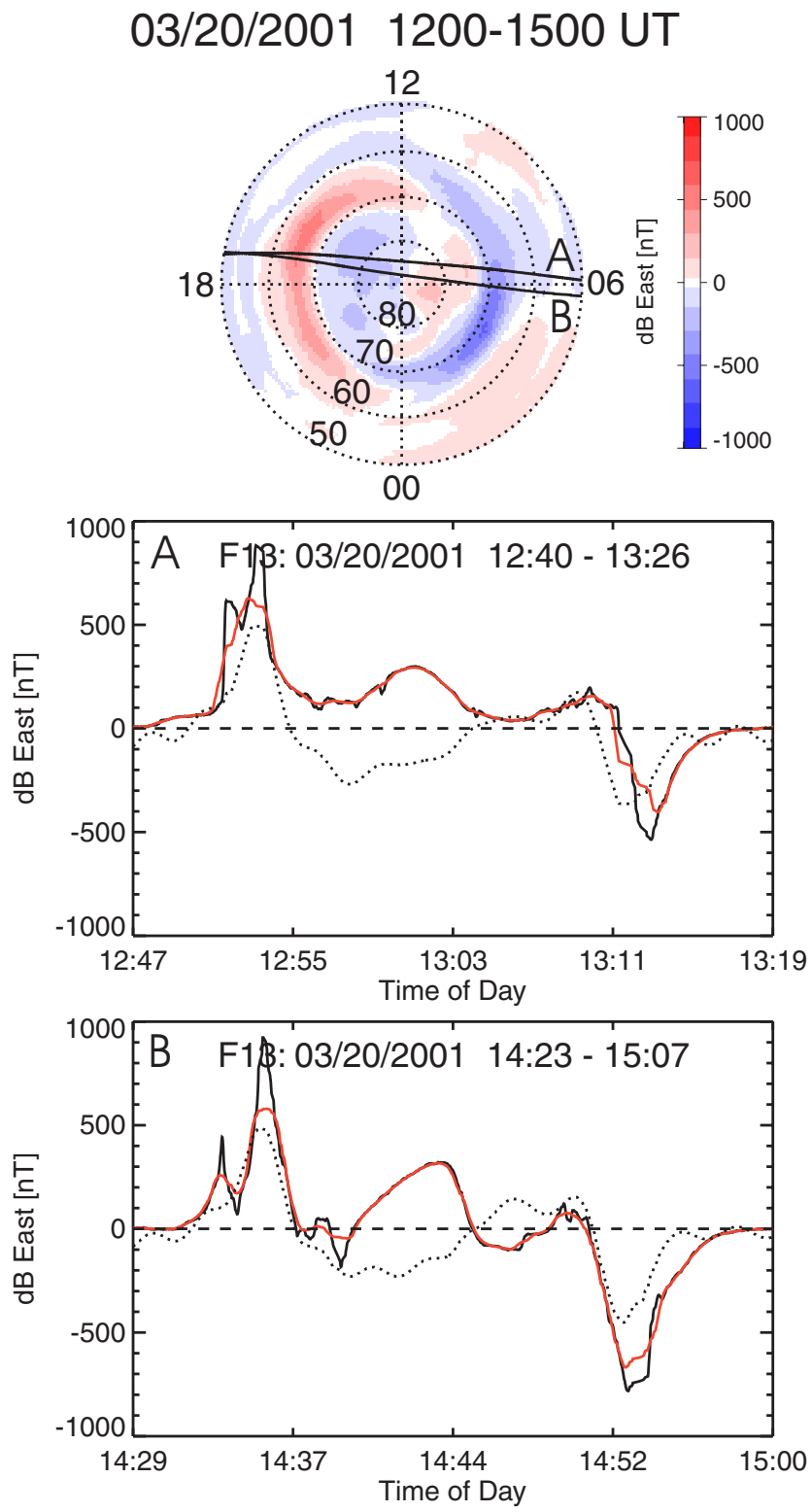

Fig. 10. Comparison of the fitted magnetic perturbations from Iridium (dotted line) with DMSP F13 observation (solid black line) during the interval 20 March 2001, 12:00-15:00 UT. The red solid line shows the $50 \mathrm{~s}$ boxcar average of the DMSP F13 data.

Two F13 passes were available for comparison during each of the two southward IMF intervals. Note that for 20 March 2001, 12:00-15:00 UT, the Iridium and DMSP observations deviate in the polar cap. During southward IMF the magnetic perturbations in the polar cap are directed primarily sunward. For three of the six Iridium orbit planes the sunward direction has a significant along-track component. The alongtrack component of the magnetic perturbations is presently not used in the spherical harmonic fit. Thus the fit may not be well-constrained in the polar cap, which is the likely cause 
for the differences in the observations in this region. We focus in our comparison on the peak magnetic perturbations observed at lower latitudes, where they are oriented predominantly in the cross-track direction of the Iridium orbit planes. Here, the peak magnetic perturbations observed by F13 are in both cases on average 1.7 times larger than those obtained from the Iridium fit. This result is consistent with previous assessments, where the Iridium peak magnetic perturbations were found to be up to $50 \%$ too low (P1, and Korth et al., 2005).

The main factor contributing to this underestimate is that the spherical harmonic fit cannot resolve features shorter than the shortest wavelength basis function. With respect to the latitude, the shortest wavelength representable by the Iridium fit corresponds to a constant angular resolution of $3.6^{\circ}$. The ionospheric grid in the simulation is irregular, leading to a dependence of the latitude resolution on both latitude and longitude. The spacing of the grid points is smallest along the noon-midnight meridian and maximizes in the dawn-dusk direction. Figure 11 shows the grid latitude separation for the noon-midnight (dotted line) and the dawn-dusk (solid line) meridian as a function of geomagnetic latitude. The figure shows that the spacing of the grid points in latitude decreases asymptotically toward the simulation inner boundary. However, poleward of $60^{\circ}$, where most of the currents discussed here are located, the change in resolution is limited to $\sim 10 \%$. The figure also shows that the latitude resolution of the simulation is higher than the one of the observations throughout this region, where the average resolution along the noon-midnight and dawn-dusk meridians is $2.1^{\circ}$ and $3.4^{\circ}$, respectively. Previous comparisons of the total current have shown that the an increase in the resolution of the simulation by a factor of 2 also increases the total current by $\sim 50 \%$. Therefore, the larger currents in the simulation may be caused in part by the higher resolution in the simulation.

The extent to which the smaller magnetic perturbations from Iridium underestimate of the Birkeland currents of the simulation can be inferred from comparison with smoothed DMSP data, where features with spatial scales smaller than simulation grid resolution are removed. The red solid lines in Figs. 9 and 10 represent $50 \mathrm{~s}$ boxcar averages of the F13 data, which are commensurate with the $3^{\circ}$ latitude resolution of the simulation grid. For both intervals presented in these figures the fitted peak magnetic perturbations are on average 0.8 times the peak magnitudes obtained from the smoothed F13 data. Therefore, the difference in resolution can account for a modest underestimate of the Birkeland currents with respect to the simulation results. However, it cannot explain the more significant differences in the magnitudes of the Birkeland currents obtained for southward IMF, suggesting that factors other than the grid resolution contribute to the discrepancy. This conclusion is supported by comparison of the Iridium magnetic perturbations for the duskward IMF interval 19 August 2003, 14:00-15:00 UT, shown in Fig. 12. In the region near the magnetic pole, the peak magnitudes of

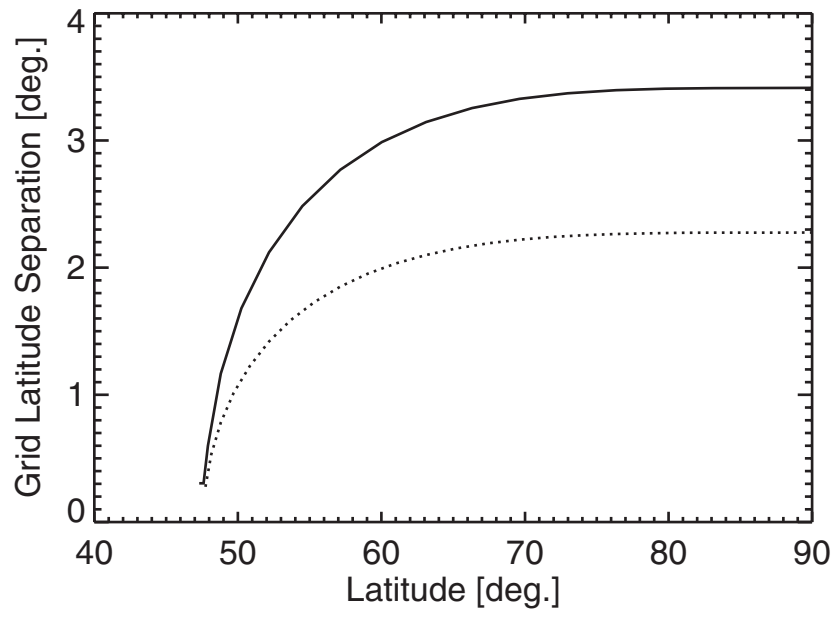

Fig. 11. Latitude separation of the grid points along the dawn-dusk (solid line) and noon-midnight (dotted line) meridian as a function of geomagnetic latitude.

the eastward magnetic perturbations from DMSP F13 and Iridium are in good agreement, and thus do not provide an explanation for why the Birkeland total currents in the simulation are 1.5 times larger for this interval than observed by Iridium.

An overestimate of the field-aligned currents in the simulation can further result from a larger than observed ionospheric conductance, or electric field, or both. To differentiate between these possibilities, we compare the LFM electric potential with in-situ observations by DMSP. Figure 13 compares a three-hour average of the LFM electric potential near $D_{s t}$ minimum from 11:30-14:30 UT on 18 August 2003 with measurements by the DMSP F13 and F15 satellites traversing the potential distribution within this period. The LFM potential distribution (Fig. 13a) exhibits a cross polar cap potential drop of $\sim 330 \mathrm{kV}$, and the axis of symmetry of the distribution is tilted toward pre-noon. Overlayed on Fig. 13a are the DMSP F13 and F15 passes, which are approximately 18:00 MLT to 06:00 MLT and 21:00 MLT to 09:00 MLT, respectively. The electric fields inferred from the driftmeter instruments are shown as dotted lines in Fig. 13b and c along with the integrated electric potential represented by the solid line. For comparison, the dashed lines show electric potentials of the simulation along the DMSP trajectories. The F13 satellite traverses close to the regions where the electric potential peaks in the simulation, observing a peak-to-peak potential drop of $170 \mathrm{kV}$. Compared to the observations, the LFM potential drop along the F13 orbit of $270 \mathrm{kV}$ is significantly larger. Assuming that the conductance estimates in the sunlit ionosphere along the F13 orbit are essentially correct in the simulation, the higher than observed electric potential implies an increased flow of electric current across the polar cap in the simulation. While F13 samples the bulk of the 
08/19/2003 1400-1500 UT
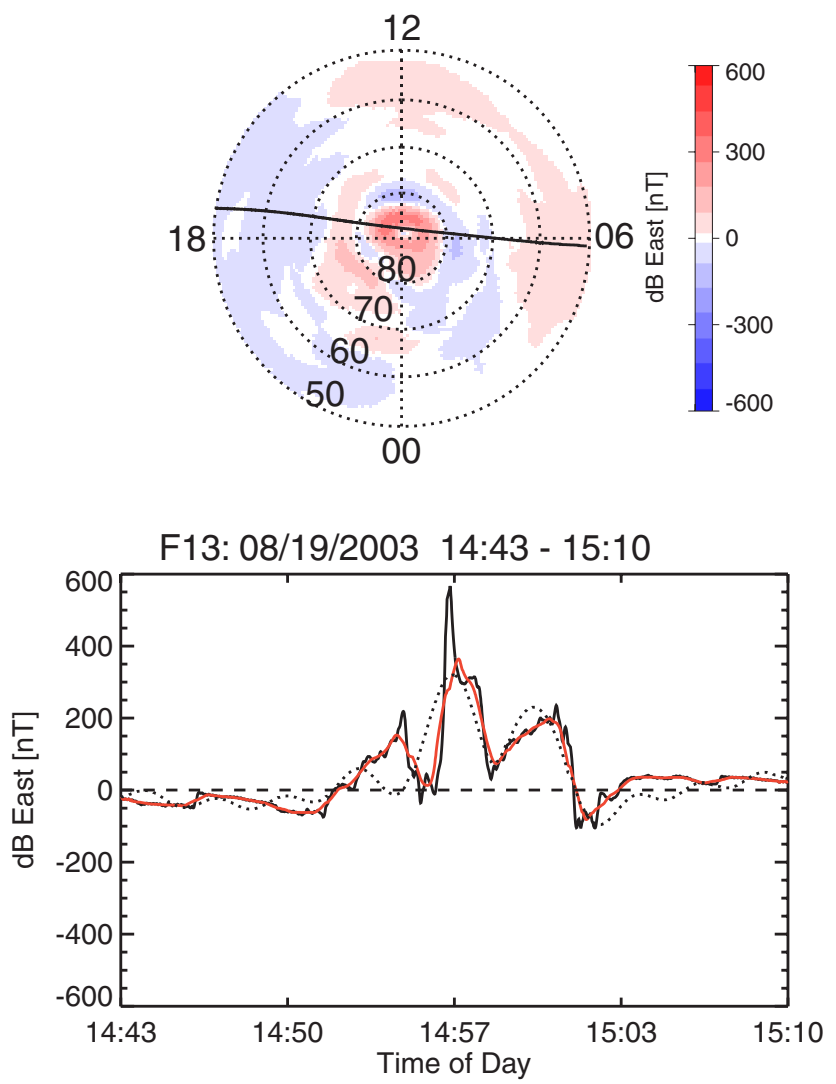

Fig. 12. Comparison of the fitted magnetic perturbations from Iridium (dotted line) with DMSP F13 observation (solid black line) during the interval 19 August 2003, 14:00-15:00 UT. The red solid line shows the $50 \mathrm{~s}$ boxcar average of the DMSP F13 data.

LFM polar cap potential drop, the F15 trajectory is oriented nearly anti-parallel to the tailward flow of plasma in the ionosphere. F15 measures a much smaller electric potential difference, indicating that the rotation of the ionospheric convection pattern with respect to the noon-midnight meridian is well captured by the model. The F15 observations further show that the electric potential develops on the nightside at latitudes lower than those obtained from the simulation, providing additional evidence that the electric field on the nightside is shorted out by the high conductivities in the MHD model.

Indications for an overestimate of the electric potential in the simulation are also found for the 20 March 2003, 12:00-15:00 UT, period. In Fig. 14, the electric potential obtained from DMSP F13 and F15 observations is compared with the simulation results along the respective satellite orbit in the same format used is Fig. 13. The F13 satellite traverses near the peaks of the LFM electric potential distribution (Fig. 14b), while the F15 trajectory (Fig. 14c) is more closely aligned with the convection "throat," where the elec-
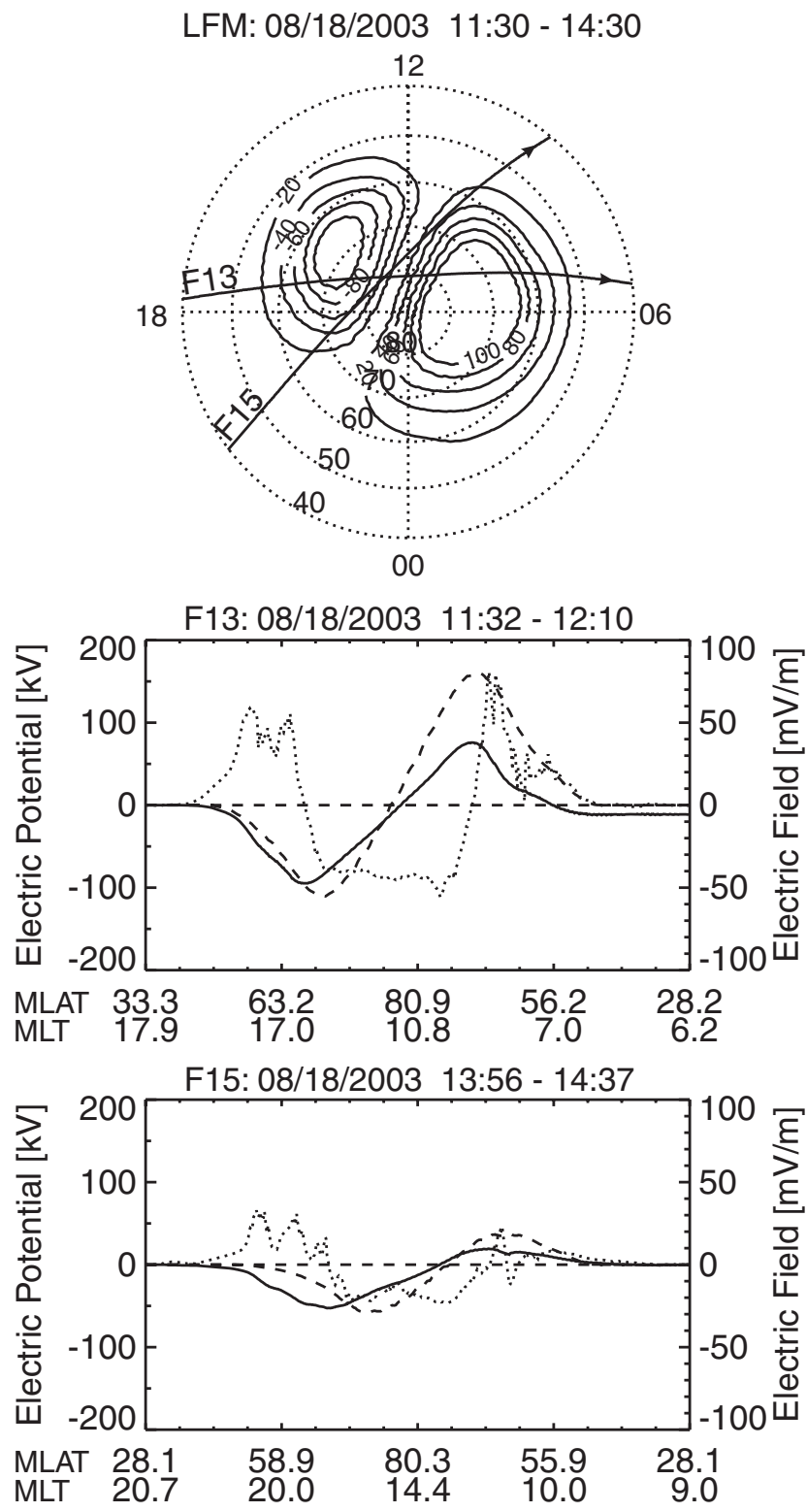

Fig. 13. Comparison of (a) the LFM electric potential distribution with observations by (b) DMSP F13 and (c) DMSP F15 on $18 \mathrm{Au}-$ gust 2003, 11:30-14:30 UT. The line plots show the DMSP electric field (dotted line) and electric potential (solid line) and the LFM electric potential interpolated along the satellite orbit.

tric potential minimizes. The electric potential drop along the F13 orbit obtained from the simulation results is $298 \mathrm{kV}$, about a factor of two larger than the $143 \mathrm{kV}$ observed by F13. The peak-to-peak difference of the electric potential along the F15 pass is smaller in both observations and simulation results, consistent with the rotation of the convection pattern toward pre-noon. 


\subsection{Simulation current closure}

The deficit of Region-2 current in the simulation suggests that the closure of current, and hence the electric field distribution in the simulation is different from the natural system. To examine the ionospheric current closure in the simulation we consider the ionospheric horizontal current. The continuity of the Birkeland currents has to be maintained in the ionosphere, so that the height-integrated current density, $\boldsymbol{J}_{\perp}$, can be calculated from the ionospheric electric field, $\boldsymbol{E}$, as well as the Pedersen and Hall conductances, $\Sigma_{\mathrm{P}}$ and $\Sigma_{\mathrm{H}}$, in the simulation:

$\boldsymbol{J}_{\perp}=\Sigma_{\mathrm{P}} \boldsymbol{E}_{\perp}-\Sigma_{\mathrm{H}}\left(\boldsymbol{E}_{\perp} \times \boldsymbol{B}\right) / B$,

where $\boldsymbol{B}$ is the magnetic field vector (Baumjohann and Treumann, 1997). By examining the current flowing through circles of constant latitude one can determine the partitioning of the Region-1 current closure in the simulation domain. The amount of current closing in the polar cap is estimated by subtracting the average inward and outward current flow across the $60^{\circ}$ MLAT latitude circle, located just equatorward of the simulated Region-1 Birkeland currents, from the average upward and downward field-aligned current found in the simulation poleward of this boundary. Calculating the current flow across the $54^{\circ}$ MLAT latitude circle, located just poleward of the zero potential boundary, shows how much of the equatorward current closure occurs via the simulation inner boundary. The closure of electric current on the simulation inner boundary is a consequence of the imposed condition that the electric field is zero equatorward of the ionospheric mapping of the inner boundary. The boundary currents therefore present a path for closure of Region-1 current that is an artefact in the sense that the closure path is prescribed as a boundary condition and effectively plays the role of a Region-2 system.

The results of this analysis show that although a significant current closes through the simulation inner boundary, the polar cap currents are dramatically over estimated in the simulation. For the 18 August 2003, 08:00-10:00 UT interval, 3.8 MA flow out and return through the $60^{\circ}$ MLAT latitude circle, accounting for about half the Region-1 currents in the simulation. Approximately 2.9 MA (or 75\%) of this current also flows across the $54^{\circ}$ MLAT latitude circle and closes on the simulation inner boundary. The current flowing across the noon-midnight meridian poleward of $60^{\circ}$ MLAT is 3.0 MA. The polar cap current in the simulation therefore exhibits nearly the same magnitude as the average Region-1 current observed by Iridium. However, the majority of the observed Region-1 currents closes at lower latitudes via Region 2 so the comparison implies an excess flow of electric current across the polar cap in the simulation. This is consistent with the comparison of DMSP potentials with the simulations presented above and therefore indicates that the discrepancy is due to a true difference in the simulation potential rather than to the conductance in the simulation.
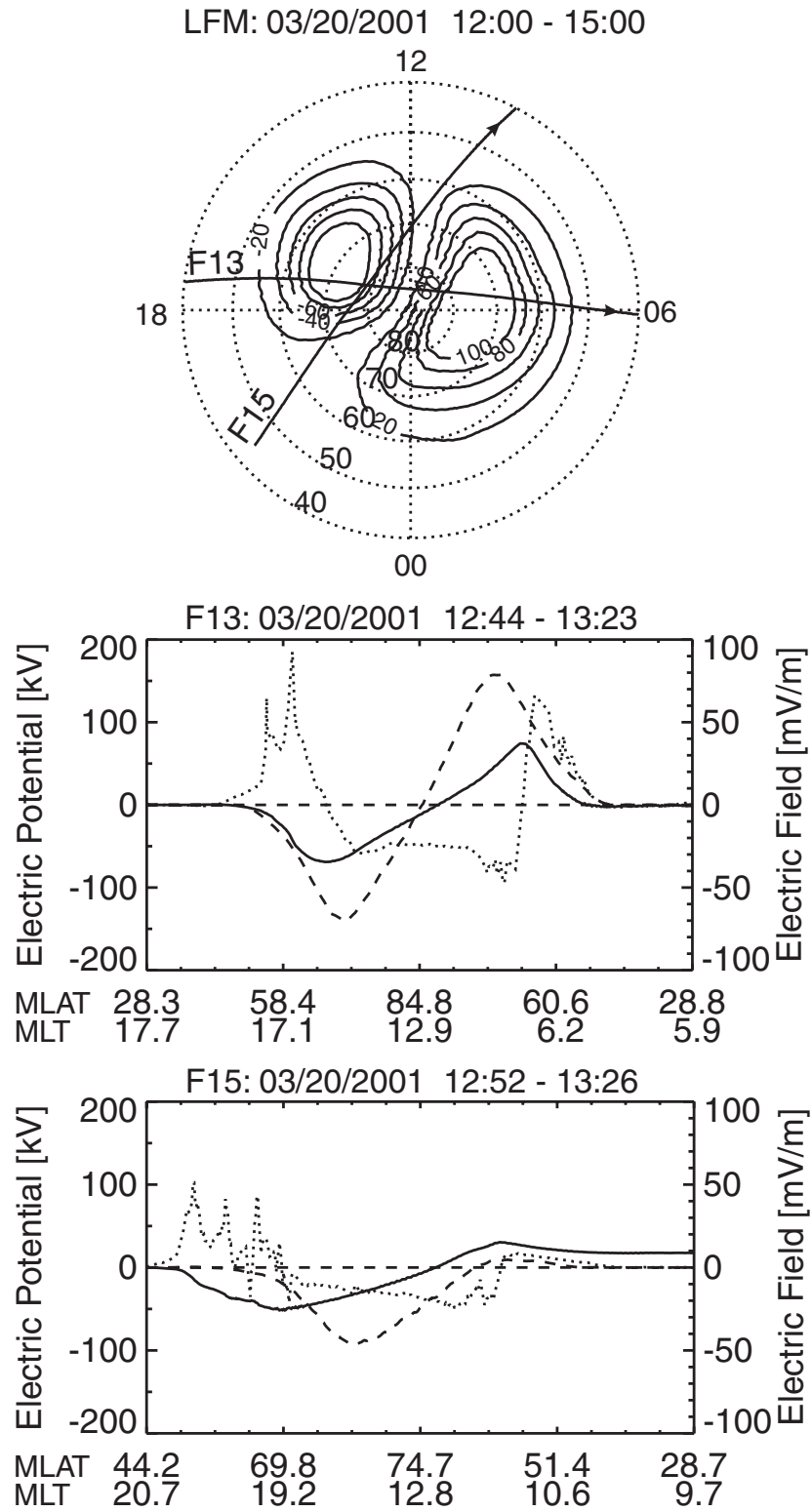

Fig. 14. Comparison of (a) the LFM electric potential distribution with observations by (b) DMSP F13 and (c) DMSP F15 on 20 March 2001, 12:00-15:00 UT. The line plots show the DMSP electric field (dotted line) and electric potential (solid line) and the LFM electric potential interpolated along the satellite orbit.

The ionospheric electric field is governed by the strength of the convection at the ionosphere, which in turn is controlled by the reconnection rate. Our results therefore suggest that the reconnection rate in the MHD model is higher than in the natural system. Similar findings have been reported for other MHD models. Raeder et al. (1998, 2001) compared ionospheric parameters obtained from a different MHD simulation code with inversion results from the Assimilative Mapping of Ionospheric Electrodynamics (AMIE) 
technique (Richmond and Kamide, 1988), which uses ground magnetometer, radar, and satellite observation to infer the conditions at the ionosphere. Consistent with our results, these authors found that while the convection pattern of their MHD model agrees with the AMIE result, the strength of the convection can be significantly different. For example, the magnitudes of the cross polar cap potential in the Raeder et al. (1998) simulation were about twice that obtained from the AMIE technique. Raeder et al. (2001) also report polar cap potentials in the simulation that are consistently higher than those of the AMIE inversions and field-aligned current densities that are up to four times larger. They concluded that the origin of the increased field-aligned currents is the large electric potential which in turn is caused by reconnection rates that are too high.

\section{Summary}

We have compared observations of the large-scale Birkeland currents by the constellation of Iridium satellites with MHD simulation results for two magnetic cloud intervals on 1719 August 2003 and 19-21 March 2001. These intervals were specifically chosen because of the slow rotation of the IMF orientation, which allows one to capture the distribution of Birkeland currents using data accumulation periods from the Iridium magnetometers. The observed Birkeland current distributions yield patterns predicted by the Reiff and Burch (1985) model, showing the classic Region-1/2 current systems for southward IMF and high-latitude NBZ currents for strongly northward IMF. Furthermore, the distributions are consistent with transformations of the ionospheric convection pattern in response to the IMF $B_{y}$, which determines the local time at which solar wind and terrestrial magnetic field lines reconnect at the dayside magnetopause. The most significant findings with respect to the comparison with the MHD simulation results are as follows:

1. The field-aligned current distributions in the MHD simulation compare qualitatively well with the observations for northward IMF, when the currents occur at high latitudes. This indicates that the MHD model adequately reproduces the convection pattern of the natural system.

2. For southward IMF, the simulation reproduced the dayside Region-1 currents generally well, although they were found to be located $\sim 5^{\circ}$ poleward of their observed latitude. Disparities in the distributions of the currents were most prominent in regions of darkness, where the conductance is dominated by particle precipitation represented by a statistical model in the simulation, and at latitudes where the Region-2 Birkeland currents of the ring current map to the ionosphere. Adequate representation of Region 2 in the MHD model requires consideration of the drift physics, which is currently work in progress (Toffoletto et al., 2004).
3. The peak field-aligned current densities and the total currents in the simulation are commonly found to exceed the observations, which is a combined effect of the simulation overestimating the ionospheric electric field and the Iridium fits underestimating the magnetic perturbations. The contribution of the marginal difference in latitude resolution to the disparities in the magnitudes of the observed Birkeland currents and the simulation results was estimated to be small. The comparison of the electric potential in the simulation with that obtained from in-situ observations of ionospheric plasma drifts by DMSP shows that the maximum potential difference in the simulation is about twice that inferred from the DMSP observations during southward IMF, implying that the reconnections rates in the simulation are too large.

Acknowledgements. We thank Iridium Satellite LLC for providing the engineering magnetometer data for scientific analysis. Furthermore, we thank the ACE MAG and SWEPAM instrument teams and the ACE Science Center for providing the ACE data. We also thank the teams of the WIND MFI and SWE instruments for providing their data for this study. Finally, we acknowledge the Center for Space Sciences at the University of Texas at Dallas and the U.S. Air Force for providing the DMSP driftmeter and magnetometer data. The research of this paper was supported by NASA grants NAG512303 and NAG510849 and NSF grant ATM-0334668.

Topical Editor M. Pinnock thanks T. Laitinen and another anonyous referee for their help in evaluating this paper.

\section{References}

Anderson, B. J., Takahashi, K., and Toth, B. A.: Sensing global Birkeland currents with Iridium engineering magnetometer data, Geophys. Res. Lett., 27, 4045-4048, 2000.

Anderson, B. J., Takahashi, K., Kamei, T., Waters, C. L., and Toth, B. A.: Birkeland currents sytem key parameters derived from Iridium observations: Method and initial validation results, J. Geophys. Res., 107, 1079, doi:10.1029/2001JA000080, 2002.

Baker, K. B. and Wing, S.: A new magnetic coordinate system for conjugate studies at high latitudes, J. Geophys. Res., 94, 91399143, 1989.

Barbosa, D. D.: An Energy Principle for High-Latitude Electrodynamics, J. Geophys. Res., 89, 2881-2890, 1984.

Baumjohann, W. and Treumann, R. A.: Basic Space Plasma Physics, Imperial College Press, London, 1997.

Burch, J. L., Reiff, P. H., Menietti, J. D., Heelis, R. A., Hanson, W. B., Shawhan, S. D., Shelley, E. G., Sugiura, M., Weimer, D. R., and Winningham, J. D.: IMF $B_{y}$-Dependent Plasma Flow and Birkeland Currents in the Dayside Magnetosphere, 1. Dynamics Explorer Observations, J. Geophys. Res., 90, 1577-1593, 1985.

Cowley, S. W. H.: Comments on the Merging of Nonantiparallel Magnetic Fields, J. Geophys. Res., 81, 3455-3458, 1976.

Crooker, N. U.: Dayside Merging and Cusp Geometry, J. Geophys. Res., 84, 951-959, 1979. 
Erlandson, R. E., Zanetti, L. J., Potemra, T. A., Bythrow, P. F., and Lundin, R.: IMF $B_{y}$ Dependence of Region 1 Birkeland Currents Near Noon, J. Geophys. Res., 93, 9804-9814, 1988.

Fedder, J. A. and Lyon, J. G.: The Earth's magnetosphere is $165 R_{E}$ long: Self-consistent currents, convection, magnetospheric structure, and processes for northward interplanetary magnetic field, J. Geophys. Res., 100, 3623-3635, 1995.

Fedder, J. A., Lyon, J. G., Slinker, S. P., and Mobarry, C. M.: Topological structure of the magnetotail as a function of interplanetary magnetic field direction, J. Geophys. Res., 100, 3613-3621, 1995a.

Fedder, J. A., Slinker, S. P., Lyon, J. G., and Elphinstone, R. D.: Global numerical simulation of the growth phase and the expansion onset for substorm observed by Viking, J. Geophys. Res., 100, $19083-19093,1995 b$.

Fedder, J. A., Slinker, S. P., and Lyon, J. G.: A comparison of global numerical simulation results to data for the January 27-28, 1992, Geospace Environment Modeling challenge event, J. Geophys. Res., 103, 14 799-14 810, 1998.

Frank, L. A., Ashour-Abdalla, M., Berchem, J., Raeder, J., Paterson, W. R., Kokubun, S., Yamamoto, T., Lepping, R. P., Coroniti, F. V., Fairfield, D. H., and Ackerson, K. L.: Observations of plasmas and magnetic fields in Earth's distant magnetotail: Comparison with a global MHD model, J. Geophys. Res., 100, 19 177-19 190, 1995.

Goodrich, C. C., Lyon, J. G., Wiltberger, M., Lopez, R. E., and Papadopoulos, K.: An overview of the impact of the January 10 11, 1997 magnetic cloud on the magnetopshere via global MHD simulation, Geophys. Res. Lett., 25, 2537-2540, 1998.

Gosling, J. T., Thomsen, M. F., Bame, S. J., and Elphic, R. C.: Plasma flow reversals at the dayside magnetopause and the origin of asymmetric polar cap convection, J. Geophys. Res., 95, 80738084, 1990.

Iijima, T. and Potemra, T. A.: Large-Scale Characteristics of FieldAligned Currents Associated With Substorms, J. Geophys. Res., 83, 599-615, 1978.

Iijima, T. and Shibaji, T.: Global Characteristics of Northward IMFAssociated (NBZ) Field-Aligned Currents, J. Geophys. Res., 92, 2408-2424, 1987.

Iijima, T., Potemra, T. A., Zanetti, L. J., and Bythrow, P. F.: LargeScale Birkeland Currents in the Dayside Polar Region During Strongly Northward IMF: A New Birkeland Current System, J. Geophys. Res., 89, 7441-7452, 1984.

Korth, H., Anderson, B. J., Wiltberger, M. J., Lyon, J. G., and Anderson, P. C.: Intercomparison of Ionospheric Electrodynamics From the Iridium Constellation With Global MHD Simulations, J. Geophys. Res., 109, A07 307, doi:10.1029/2004JA010428, 2004.

Korth, H., Anderson, B. J., Frey, H. U., and Waters, C. L.: HighLatitude Electromagnetic and Particle Energy Flux During an Event With Sustained Strongly Northward IMF, Ann. Geophys., 23, 999-999, 2005, http://www.ann-geophys.net/23/999/2005/.

Lepping, R. P., Acuna, M. H., Burlaga, L. E., Farrell, W. M., Slavin, J. A., Schatten, K. H., Mariani, F., Ness, N. F., Neubauer, F. M., Whang, Y. C., Byrnes, J. B., Kennon, R. S., Panetta, P. V., Scheifele, J., and Worley, E. M.: The WIND magnetic field investigation, Space Sci. Rev., 71, 207-229, 1995.

Lopez, R. E., Goodrich, C. C., Wiltberger, M., and Lyon, J. G.:
Simulation of the March 9, 1995 substorm and initial comparison to data, in: Geospace Mass and Energy Flow: Results from the International Solar-Terrestrial Physics Program, edited by: Horwitz, J. L., Gallagher, D. L., and Peterson, W. K., Geophysical Monograph 104, pp. 237-245, 1998.

Lyon, J. G., Lopez, R. E., Goodrich, C. C., Wiltberger, M., and Papadopoulos, K.: Simulation of the March 9, 1995, substorm: Auroral brightening and the onset of lobe reconnection, Geophys. Res. Lett., 25, 3039-3042, 1998.

Lyon, J. G., Fedder, J. A., and Mobarry, C. M.: The Lyon-FedderMobarry (LFM) global MHD magnetospheric simulation code, J. Atmos. Solar-Terr. Phys., 66, 1333-1350, 2004.

Maezawa, K.: Magnetospheric Convection Induced by the Positive and Negative Z Components of the Interplanetary Magnetic Field: Quantitative Analysis Using Polar Cap Magnetic Records, J. Geophys. Res., 81, 2289-2303, 1976.

Massetti, S.: Antiparallel magnetic merging signatures during IMF $B_{y} \gg 0$ : longitudinal and latitudinal cusp aurora bifurcations, Ann. Geophys., 24, 2299-2311, 2006,

http://www.ann-geophys.net/24/2299/2006/.

McComas, D. J., Bame, S. J., Barker, P. L., Feldman, W. C., Phillips, J. L., Riley, P., and Griffee, J. W.: Solar Wind Electron Proton Alpha Monitor (SWEPAM) for the Advanced Composition Explorer, Space Sci. Rev., 86, 563-612, 1998.

Mobarry, C. M., Fedder, J. A., and Lyon, J. G.: Equatorial plasma convection from global simulations of the Earth's magnetosphere, J. Geophys. Res., 101, 7859-7874, 1996.

Moore, T. E., Fok, M.-C., and Chandler, M. O.: The dayside reconnection X line, J. Geophys. Res., 107, 1332, doi:10.1029/ 2002JA009381, 2002.

Ogilvie, K. W., Chornay, D. J., Fritzenreiter, R. J., Hunsaker, F., Keller, J., Lobell, J., Miller, G., Scudder, J. D., Sittler, E. C., Torbert, R. B., Bodet, D., Needell, G., Lazarus, A. J., Steinberg, J. T., Tappan, J. H., Mavretic, A., and Gergin, E.: SWE, a comprehensive plasma instrument for the WIND spacecraft, Space Sci. Rev., 71, 55-77, 1995.

Ohtani, S., Potemra, T. A., Newell, P. T., Zanetti, L. J., Iijima, T., Watanabe, M., Blomberg, L. G., Elphinstone, R. D., Murphree, S., Yamauchi, M., and Woch, J. G.: Four large-scale field-aligned current systems in the dayside high-latitude region, J. Geophys. Res., 100, 137-153, 1995.

Onsager, T. G. and Fuselier, S. A.: The location of magnetopause reconnection for northward and southward interplanetary magnetic field, in: Solar System Plasmas in Space and Time, edited by: Burch, J. L. and White, J. H., Geophysical Monograph 84, p. 183, American Geophysical Union, Washington, 1994.

Raeder, J., Berchem, J., Ashour-Abdalla, M., Frank, L. A., Paterson, W. R., Ackerson, K. L., Kokubun, S., Yamamoto, T., and Slavin, J. A.: Boundary layer formation in the magnetotail: Geotail observations and comparison with a global MHD simulation, Geophys. Res. Lett., 24, 951-954, 1997.

Raeder, J., Berchem, J., and Ashour-Abdalla, M.: The Geospace Environment Modeling Grand Challenge: Results from a Global Geospace Circulation Model, J. Geophys. Res., 103, $14787-$ 14797, 1998.

Raeder, J., McPherron, R. L., Frank, L. A., Kokubun, S., Lu, G., Mukai, T., Paterson, W. R., Sigwarth, J. B., Singer, H. J., and Slavin, J. A.: Global simulation of the Geospace Environment Modeling substorm challenge event, J. Geophys. Res., 106, 381- 
395, 2001

Reiff, P. H. and Burch, J. L.: IMF $B_{\mathrm{y}}$-Dependent Plasma Flow and Birkeland Currents in the Dayside Magnetosphere, 2. A Global Model for Northward and Southward IMF, J. Geophys. Res., 90, 1595-1609, 1985.

Richmond, A. D. and Kamide, Y.: Mapping Electrodynamic Features of the High-Latitude Ionosphere From Localized Observations: Technique, J. Geophys. Res., 93, 5741-5759, 1988.

Ridley, A. J., Zeeuw, D. L. D., and Gombosi, T. I.: Using the steady state MHD results to predict the global state of the magnetosphere-ionosphere system, J. Geophys. Res., 106, $30067-30076,2001$.

Ridley, A. J., Hansen, K. C., Toth, G., Zeeuw, D. L. D., Gombosi, T. I., and Powell, K. G.: University of Michigan MHD results of the Geospace Global Circulation Model metrics challenge, J. Geophys. Res., 107, 1290, doi:10.1029/JA000253, 2002.

Russell, C. T.: The configuration of the magnetosphere, in: Problems in Magnetospheric Physics, edited by: Dyer, E. R., pp. 116, National Academy of Science, Washington, 1972.

Sandholt, P. E., Farrugia, C. J., and Denig, W. F.: Detailed dayside auroral morphology as a function of local time for southeast IMF orientation: implications for solar wind-magnetosphere coupling, Ann. Geophys., 22, 3537-3560, 2004, http://www.ann-geophys.net/22/3537/2004/.

Slinker, S. P., Fedder, J. A., and Chen, J.: Global MHD simulation of the magnetosphere and ionosphere for 1930-2330 UT on November 3, 1993, J. Geophys. Res., 103, 26 243-26 250, 1998.

Slinker, S. P., Fedder, J. A., Emery, B. A., Baker, K. B., Lummerzheim, D., Lyon, J. G., and Rich, F. J.: Comparison of global MHD simulations with AMIE simulations for the events of May 19-20, 1996, J. Geophys. Res., 104, 28 379-28 395, 1999.
Smith, C. W., L'Heureux, J., Ness, N. F., Acuña, M. H., Burlaga, L. F., and Scheifele, J.: The ACE Magnetic Fields Experiment, Space Sci. Rev., 86, 613-632, 1998.

Toffoletto, F. R., Sazykin, S., Spiro, R. W., Wolf, R. A., and Lyon, J. G.: RCM meets LFM: Initial results of one-way coupling, J. Atmos. Solar-Terr. Phys., 66, 1361-1370, 2004.

Vennerstrøm, S., Moretto, T., Rastätter, L., and Raeder, J.: Fieldaligned currents during northward interplanetary magnetic field: Morphology and causes, J. Geophys. Res., 110, A06 205, doi: 10.1029/2004JA010802, 2005.

Waters, C. L., Anderson, B. J., and Liou, K.: Estimation of global field aligned currents using the Iridium System magnetometer data, Geophys. Res. Lett., 28, 2165-2168, 2001.

Waters, C. L., Anderson, B. J., Greenwald, R. A., Barnes, R. J., and Ruohoniemi, J. M.: High Latitude Poynting Flux From Combined Iridium and SuperDARN Data, Ann. Geophys., 22, 28612875, 2004, http://www.ann-geophys.net/22/2861/2004/.

Wiltberger, M., Pulkkinen, T. I., Lyon, J. G., and Goodrich, C. C.: MHD simulation of the magnetotail during the December 10, 1996, substorm, J. Geophys. Res., 105, 27 649-27 663, 2000.

Zanetti, L. J., Potemra, T. A., Iijima, T., Baumjohann, W., and Bythrow, P. F.: Ionospheric and Birkeland Current Distributions for Northward Interplanetary Magnetic Field: Inferred Polar Convection, J. Geophys. Res., 89, 7453-7458, 1984. 